ESAIM: M2AN 47 (2013) 1553-1582

DOI: $10.1051 / \mathrm{m} 2 \mathrm{an} / 2013075$
ESAIM: Mathematical Modelling and Numerical Analysis

www.esaim-m2an.org

\title{
ERROR ESTIMATES FOR THE COUPLED CLUSTER METHOD*
}

\author{
Thorsten RohweddeR ${ }^{1}$ And Reinhold Schneider ${ }^{1}$
}

\begin{abstract}
The Coupled Cluster (CC) method is a widely used and highly successful high precision method for the solution of the stationary electronic Schrödinger equation, with its practical convergence properties being similar to that of a corresponding Galerkin (CI) scheme. This behaviour has for the discrete CC method been analyzed with respect to the discrete Galerkin solution (the "full-CI-limit") in [Schneider, 2009]. Recently, we globalized the CC formulation to the full continuous space, giving a root equation for an infinite dimensional, nonlinear Coupled Cluster operator that is equivalent the full electronic Schrödinger equation [Rohwedder, 2011]. In this paper, we combine both approaches to prove existence and uniqueness results, quasi-optimality estimates and energy estimates for the CC method with respect to the solution of the full, original Schrödinger equation. The main property used is a local strong monotonicity result for the Coupled Cluster function, and we give two characterizations for situations in which this property holds.
\end{abstract}

Mathematics Subject Classification. 65Z05, 81-08, 70-08.

Received July 6, 2011. Revised November 26, 2012.

Published online August 26, 2013.

\section{INTRODUCTION AND OVERVIEW}

More than 80 years after Paul Dirac stated that "the underlying physical laws necessary for the mathematical theory of a large part of physics and the whole of chemistry are [...] completely known" [31], the development of "approximate practical methods of applying quantum mechanics", demanded by Dirac in the same breath, is still a highly active field of research at the crossroads of physics, chemistry, applied mathematics and computer science. After the development of modern day computer capacities has seen a phase of almost exponential growth at the end of the last century, calculations of theoretical chemistry and molecular physics have become competitive with practical experiments in the first place, or at least often allow useful predictions of empirical parameters that can assist practical investigations. On the other hand, the solution of the governing equation of quantum mechanics, the molecular Schrödinger equation, is an exceedingly high-dimensional and thus computationally demanding problem, while at the same time, an extremely high accuracy is needed in order to obtain results to be utilized in practice, so that small to medium-sized quantum chemical problems still push the limits of commonly available computational resources.

Keywords and phrases. Quantum chemistry, electronic Schrödinger equation, coupled Cluster method, numerical analysis, nonlinear operator equation, quasi-optimality, error estimators.

* This work has been supported by the DFG SPP programs 1145 and 1324.

1 Sekretariat MA 5-3, Institut für Mathematik, TU Berlin, Straße des 17. Juni 136, 10623 Berlin, Germany.

rohwedder@math . tu-berlin.de 
The electronic Schrödinger equation. When interested in molecular properties that are mainly due to the electronic structure, or as a part of an alternating computation of electronic structure and the motion of the cores within them based on the Born-Oppenheimer approximation [17,62], a problem of major concern is computation the electronic ground state $\underline{\Psi}$ of the system, describing the probability distribution of $N$ electrons in the fixed field induced by the nuclei. It can be computed by solution of the stationary electronic Schrödinger equation

$$
H \underline{\Psi}=E^{*} \underline{\Psi}
$$

a weak operator eigenvalue equation for the lowest eigenvalue $E^{*}$ of the Hamiltonian $H: \mathbb{H}^{1} \rightarrow \mathbb{H}^{-1}$ of the system as defined on the space $\mathbb{H}^{1}$ of antisymmetric functions of finite kinetic energy, with $H$ itself completely given by the charges and positions of the fixed nuclei (see (2.4)). To efficiently treat the variety of practical problems covered by (1.1), it is indispensable to design highly problem-adapted methods and algorithms that balance the available computational resources against the respective required accuracy. These prerequisites have lead to a "zoo" of well-developed and often extremely sophisticated methods and algorithms for (1.1), cf. also the corresponding entries in the recent encyclopedia [20] for their mathematical formulations: A common approach is the formulation as the minimization problem

$$
\underline{\Psi}=\operatorname{argmin}_{\Psi \in \mathbb{H}^{1}} \frac{\langle H \Psi, \Psi\rangle}{\langle\Psi, \Psi\rangle},
$$

followed by a restriction of the admitted solution space $\mathbb{H}^{1}$ to a subspace or -manifold. Minimization over antisymmetrized rank-1-approximations, for instance, leads to the well-known Hartree-Fock ansatz [23, 43, 58, 61], or, with an empirically adopted functional, to the successful method of Density Functional Theory (DFT) [1, $32,70,81]$. These two are probably most important methods for the qualitative study of larger systems.

The Configuration Interaction (CI) method. If the accuracy provided by HF or DFT is not sufficient for the problem under consideration, this is then followed by a usually computationally more demanding postHartree-Fock calculation, which uses the computed rank-one solution $\Psi_{0}$ as reference solution for the computation of a refined discrete solution $\underline{\Psi}_{D}=\Psi_{0}+\Psi_{D}^{*}$ with an orthogonal correction $\Psi_{D}^{*}$. A straightforward such approach is a Galerkin ansatz, known as full Configuration Interaction ansatz in the quantum chemists' community, yielding the eigenvalue problem

$$
\left\langle H\left(\Psi_{0}+\Psi_{D}^{*}\right), \Psi_{D}\right\rangle=E\left\langle\Psi_{0}+\Psi_{D}^{*}, \Psi_{D}\right\rangle \quad \text { for all } \Psi_{D} \in \mathbb{H}_{D}^{1}
$$

Denoting by $N$ the number of particles to be modelled by $\Psi_{D}$, the ansatz space $\mathbb{H}_{D}^{1}$ is a "full" discrete tensor product space, spanned by all possible choices of $N$-electron Slater determinants (i.e., antisymmetric basis functions, see Sect. 2 for a detailed definition) that can be formed from a previously chosen set of $K>N$ oneparticle functions $\chi_{I} \in H^{1}\left(\mathbb{R}^{3} \times\left\{ \pm \frac{1}{2}\right\}\right)$. $\mathbb{H}_{D}^{1}$ is of the usually vast dimension $\left(\begin{array}{l}K \\ N\end{array}\right)$. Therefore, to push practically relevant problems into the range of computability, it has to be truncated to some subspace $\mathbb{H}_{d}^{1} \subseteq \mathbb{H}_{D}^{1}$, usually done according to selection rules based on the excitation level of the basis function [43], resulting in a "truncated CI" method. If the reference $\Psi_{0}$ is sufficiently good, it often suffices to choose only lower excitations of $\Psi_{0}$ as a basis for the ansatz space, viz., basis functions that differ from $\Psi_{0}$ only in in, say, two to four one-particle functions (see Sect. 2(ii)), to obtain practicable results on the one hand and stay in a range of computational feasibility on the other. The CI approach shares the enjoyable analytic properties of the Galerkin method applied to symmetric eigenvalue problems [22]: The CI method satisfies the Rayleigh-Ritz variational principle, and if the lowest eigenvalue $E^{*}$ of $H$ is simple, it is well-known that quasi-optimality estimates for the approximation error of the discrete solution $\underline{\Psi}_{d}$ with respect to the real solution $\underline{\Psi}=\Psi_{0}+\Psi^{*}$ of (1.1), (1.2) hold, being of the form

$$
\left\|\underline{\Psi}_{d}-\underline{\Psi}\right\| \lesssim d\left(\mathbb{H}_{d}^{1}, \underline{\Psi}\right), \quad E\left(\underline{\Psi}_{d}\right)-E^{*} \lesssim\left\|\underline{\Psi}_{d}-\underline{\Psi}\right\|^{2} \lesssim d\left(\mathbb{H}_{d}^{1}, \underline{\Psi}\right)^{2} .
$$


In the most basic of these estimates, the inverse of the spectral gap $\gamma^{*}$ between $E^{*}$ and the rest of the spectrum enters as a constant and distances are measured with respect to the $\mathbb{H}^{1}$-norm, see also $[6,22,88]$ for more refined estimates.

In contrast to these nice theoretical properties is a major practical shortcoming of CI: It seems to be rather undoubted in the quantum chemist's community that a method should have the property of being size-consistent $[10,12,43,67]$, meaning, formulated in a somewhat loose fashion, the following: Suppose that the Hamiltonian $H$ can be written as sum of two non-interacting ${ }^{2}$ Hamiltonians, $H=H_{A}+H_{B}$. Then a method is size-consistent if the energy computed by that method for $H$ is the same as the sum of the energies computed by the method applied to $H_{A}$ and $H_{B}$ separately. Unfortunately, the truncation step from the "full CI" space $\mathbb{H}_{D}^{1}$ to the "truncated CI space" $\mathbb{H}_{d}^{1}$ - making CI computations computationally feasible in the first place results in a non-size-consistent ansatz, see [43]. This deficiency leads e.g. to inappropriate dissociation curves in the description of reaction mechanisms and renders the CI method useless in many practical applications.

The Coupled Cluster approach. The Coupled Cluster (CC) approach, introduced in the field of atomic physics $[25,26,51,86]$ and later used on problems of quantum chemistry (see [24]), remedies this shortcoming by replacing the linear ansatz of full CI by an equivalent exponential parametrization of the sought solution $\underline{\Psi}_{D}$, i.e. it reformulates

$$
\underline{\Psi}_{D}=\underbrace{\Psi_{0}+\Psi_{D}^{*}=\Psi_{0}+\sum_{\mu \in \mathcal{M}_{D}} c_{\mu} \Psi_{\mu}}_{\text {CI ansatz }}=\underbrace{\mathrm{e}^{T\left(t_{D}\right)} \Psi_{0}}_{\text {CC ansatz }}
$$

for a coefficient vector $t_{D}=\left(t_{\mu_{D}}\right)_{\mu_{D} \in \mathcal{M}_{D}}$, determining a corresponding cluster operator $T\left(t_{D}\right)$ (see Sect. 2 for details). After additionally inserting $\mathrm{e}^{-T\left(t_{D}\right)}$ (without affecting the solution, see [79]), this ansatz leads to a nonlinear equation

$$
f\left(t_{D}\right)=\left\langle\Psi_{\mu_{D}}, \mathrm{e}^{-T\left(t_{D}\right)} H \mathrm{e}^{T\left(t_{D}\right)} \Psi_{0}\right\rangle=0 \quad \text { for all } \Psi_{\mu_{D}} \perp \Psi_{0}
$$

for $t_{D}$, and $\underline{\Psi}_{D}=\mathrm{e}^{T\left(t_{D}\right)} \Psi_{0}$ equals the full CI-solution of (1.3). As (1.3), (1.5) is normally computationally infeasible and therefore projected to a subset $\mathcal{M}_{d} \subseteq \mathcal{M}_{D}$ of the full CI space, resulting in a projected Coupled Cluster equation in which $t_{d}=\left(t_{\mu_{d}}\right)_{\mu_{d} \in \mathcal{M}_{d}}$ has to be computed such that

$$
f\left(t_{d}\right)=\left\langle\Psi_{\mu_{d}}, \mathrm{e}^{-T\left(t_{d}\right)} H \mathrm{e}^{T\left(t_{d}\right)} \Psi_{0}\right\rangle=0 \quad \text { for all } \Psi_{\mu_{d}} \perp \Psi_{0} .
$$

Equations (1.5), (1.6) constitute the central equations of the (finite-dimensional) Coupled Cluster ansatz; for deeper insight, $c f$. $[11,52]$ and the abundance of references given therein, as well as the article [16] for a scope on the applications in physics; for some recent developments, see, e.g., [14, 19, 21, 34, 47, 53, 64, 65, 83]. Solution of the nonlinear equation (1.6) is a rather complicated matter of its own owed to the incorporation of antisymmetry constraints imposed on the solution space $\mathbb{H}^{1}$. Nevertheless, practical difficulties arising here have been overcome during the last decades; in particular, the operator $\mathrm{e}^{-T\left(t_{d}\right)} H \mathrm{e}^{T\left(t_{d}\right)}$ can be expanded in the so-called Baker-Campbell-Hausdorff series, which terminates due to the structure of the Hamiltonian $H . f\left(t_{d}\right)$ turns out to be of fourth-order in the coefficients $t_{\mu_{d}}$ of $t_{d}$, and thus can be evaluated exactly within a given finite basis set by rather advanced algorithms, ( $c f$. Sect. 5 for more practical remarks).

CC versus CI; "degenerate" situations. Compared to the CI ansatz, some important features of CC are the following:

$\triangleright$ The truncation step from (1.5) to (1.6) gives a set of equations that is (in contrast to truncated CI) still sizeconsistent, and from the solution of (1.6), the corresponding size-consistent CC energy $E_{d}$ can be computed, see [43], Section 13.3 for a rigorous proof. This property of CC is indeed the main motivation for usage of the CC method in place of the CI method in applications.

\footnotetext{
${ }^{2}$ The formal definition of "non-interacting" in a mathematically rigorous setting is that the creation and annihilation operators contained in $H_{A}$ and $H_{B}$ anticommute, see the appendix for the definition of these quantities.
} 
$\triangleright$ In contrast to CI, CC is non-variational, i.e., the discrete energy $E_{d}$ is no longer an upper bound for the ground state energy $E^{*}$. This may be seen as a disadvantage of $\mathrm{CC}$, but it seems to be generally accepted that the loss of this property is a minor trade-off against that of size-consistency.

Concerning the characterization of situations where CI/CC can be applied with reasonable computational power to give accurate results, $\mathrm{CC}$ of a certain truncation level shares very much the strengths and weaknesses of the corresponding CI method, and the characterization of situations to which CI/CC can be applied are in fact in the literature often treated on the same theoretical footing.

$\triangleright$ On the one hand, when applied to well-behaved examples, typically closed shell molecules at geometries near the equilibrium state (being an important standard situation in computation of uncharged molecules), inclusion of only relatively few basis functions in the ansatz (1.6) is often sufficient. The Coupled Cluster method then usually outperforms the CI method of corresponding scaling, especially for larger examples, see e.g. $[27,44,50]$. In the variant of the $\operatorname{CCSD}(\mathrm{T})$ method [75], which can be applied to small to medium-sized molecules with reasonable computational effort, $\mathrm{CC}$ then often provides results which are within the error bars of corresponding practical experiments [55], which is why $\operatorname{CSSD}(\mathrm{T})$ is often referred to as the "golden standard of quantum chemistry". When computing different levels of approximation $\underline{\Psi}_{d}$ to a discrete full-CI solution $\underline{\Psi}_{D}$ for well-behaved examples, the energies are often observed to converge quadratically with respect to the energy error of the cluster amplitude vectors $\left\|t_{d}-t_{D}\right\|_{\mathbb{V}}$ in analogy to the right equation of the CI estimate (1.4).

$\triangleright$ On the other hand, CC fails on the same problem classes as the corresponding CI methods: E.g., for the description of dissociation of molecules, of open-shell systems, of metallic or quasi-metallic systems and systems far from their equilibrium geometry, application of CC/CI (as of essentially any correlation method based on a single reference, see, e.g., reference [76]) is well-known to be problematic. In such cases, calculations may have to include a large number of basis functions from $\mathbb{B}$, so that essentially, almost a full-CI calculation within a large basis set has to be performed, or the method may even fail to converge. See, e.g., the examples in $[38,39]$, and also [43] for an instructive example.

To sum up, the CC method has due to its favourable properties of size-consistency and due to an often quite favourable convergence behaviour over the CI method become the probably most widely applied tool in the calculation of ground state solutions of the stationary $N$-electron Schrödinger equation when high-accuracy results are demanded, i.e., when accuracies provided by HF or DFT are not sufficient. On the other hand, situations in which CC fails - as described in the latter point - are called degenerate or quasi-degenerate situations in the quantum chemistry literature. Although the terminology "degeneracy" is used somewhat ambiguous in the quantum chemists' community, situations where the failure of CC/CI is observed may be differentiated into two cases, see, e.g., [69]: Those where a single determinant $\Psi_{0}$ does not describe the system sufficiently well ("static" or "non-dynamic correlation", [48]), and those where spectral gaps are small ("system degeneracy"), as in metallic systems or during the process of breaking of bonds, see, e.g., [38,39,43]. Both situations may occur together, but either one may negatively affect the convergence of CC [69].

Development of methods for the generic treatment of such degenerate situations is a very important, unfortunately rather open question of quantum chemistry, and there is a whole variety of active efforts to obtain versions of CC to deal with these problems, see, e.g., the recent [19] for various multi-reference approaches or [73] for other alternatives in the spirit of the CC ansatz.

CC from the perspective of numerical analysis. The vast amount of publications on CC is contrasted by a scarcity of theoretical results underpinning this behaviour in terms of a numerical analysis. To the authors' knowledge, the only existence and uniqueness results are those for the discrete equations given in [82], while there are no results on the convergence of the exact, continuous equations; also, the similarity to the convergence behaviour of the CI method has not been investigated from a rigorous mathematical viewpoint. A first error bound for the convergence of the energies to the discrete "full-CI energy" was given in [50], where Werner 
Kutzelnigg derives the estimate

$$
\left|E_{d}-E_{D}\right| \lesssim\left\|\underline{\Psi}_{d}-\underline{\Psi}_{D}\right\|\left\|\underline{\Psi}_{d}\right\| \sim\left\|t_{d}-t_{D}\right\|\left\|t_{d}\right\| .
$$

Comparing this with what is known for the CI method (1.4), this estimate is unsatisfactory in view of the similar practical convergence behaviour of the CC method. In the analysis of the CC method, two peculiarities of the classical formulation of the CC approach have to be taken into account:

(a) CC, in its classical formulation, is a finite dimensional theory as reformulation of the full CI-approach, that is, as a nonlinear equation for coefficients in a fixed, discretized basis set. In a previous publication [79], we showed that if the reference $\Psi_{0}$ is not orthogonal to the solution $\underline{\Psi}$, the original full electronic Schrödinger equation (1.1) can also be reformulated equivalently as a root equation for a continuous Coupled Cluster function

$$
f: \mathbb{V} \rightarrow \mathbb{V}^{\prime}, \quad f(t):=\left(\left\langle\Psi_{\mu}, \mathrm{e}^{-T(t)} H \mathrm{e}^{T(t)} \Psi_{0}\right\rangle\right)_{\mu \in \mathcal{M}},
$$

where $f$ is defined on a coefficient space $\mathbb{V}$ corresponding to the full continuous space $\mathbb{H}^{1}$, and that the discrete $\mathrm{CC}$ equations (1.6) can be viewed as Galerkin discretizations of (1.8) (see Sect. 2 for a recap). Using these results, we may thus rely on the machinery of operator analysis to obtain direct quasi-optimality estimates with respect to the solution $t^{*}$ of (1.8), corresponding to the full solution $\underline{\Psi}=\mathrm{e}^{T\left(t^{*}\right)} \Psi_{0}$.

(b) CC, in its classical formulation, is non-variational, so that one cannot expect estimates for the CC energy that take the simple form as for CI, (1.4). Instead, an approach put forward by Rannacher and coworkers $[9,13]$, tailored for goal-oriented estimates for non-symmetric eigenvalue problems and non-symmetric constrained minimization problems, offers the appropriate tool. Here, a dual solution $z^{*}$ and its discrete counterpart $z_{d}$ enter the estimates. For the discrete case, we already used this approach in [82] to show that

$$
\left|E_{d}-E_{D}\right| \lesssim\left(\left\|t_{d}-t_{D}\right\|_{\mathbb{V}_{d}}+\left\|z_{d}-z_{D}\right\|_{\mathbb{V}_{d}}\right)^{2}
$$

holds for the discrete energies $E_{d}, E_{D}$ if the CC approach is based on eigenfunctions of a discrete Fock operator. Alas, this condition excludes many of the more sophisticated CC approaches; also, all estimates in [82] are given with respect to the full CI (discrete) solution, and then connected to the full, continuous solution of (1.1) using the quasi-optimality of the CI-method and certain uniformity conditions, and we will also avoid this detour here.

Contributions and assumptions of this work; outline. We will in this work prove that also from a theoretical point of view, the (single-reference) CC method behaves similar to the (single-reference) CI method: Under conditions that parallel those for the results (1.4) for the CI method, we will prove certain existence and uniqueness statements for the continuous as well as for the discrete CC equation, analogous to those for the Galerkin method. Also, as the result with probably the most practical significance, we prove quasi-optimal convergence estimates for the CC amplitudes and the CC energy towards the respective full solution of (1.1), that is,

$$
\begin{gathered}
\left\|t_{d}-t^{*}\right\|_{\mathbb{V}} \lesssim d\left(\mathbb{V}_{d}, t^{*}\right) \\
\left|E\left(t_{d}\right)-E^{*}\right| \lesssim\left(\left\|t_{d}-t^{*}\right\|_{\mathbb{V}}+\left\|z_{d}-z^{*}\right\|_{\mathbb{V}}\right)^{2} \lesssim\left(\max \left\{d\left(\mathbb{V}_{d}, t^{*}\right), d\left(\mathbb{V}_{d}, z^{*}\right)\right\}\right)^{2}
\end{gathered}
$$

In these estimates, $\|t\|_{\mathbb{V}}$ denotes a weighted $\ell_{2}$-norm on the coefficient space $\mathbb{V}$ as above, equivalent to the $\mathbb{H}^{1}$ norm of $\sum_{\mu \in \mathcal{M}} t_{\mu} \Psi_{\mu}$, and $z_{d}, z^{*}$ are solutions of a dual problem entering through the non-variational nature of the CC equations ( $c f$. Sect. 4). The energy estimates (1.11) will be based on the dual weighted residual approach due to Rannacher et al. [9,13], applied to the Coupled Cluster Lagrangian as used in linear response theory $[4,29,42]$. To our knowledge, our work is the first error analysis for Coupled Cluster based on the primal/dual residual approach for non-variational problems, considerably improving the previously known estimates (1.7).

The mathematical key feature of the CC equations enabling the above results is a local strong monotonicity property of the continuous CC function (1.8) at the solution $t^{*}$. Although the local version that we formulated 
does not seem to be common in the literature, modifications of arguments from standard operator theory then apply to interpret the discrete CC equations (1.6) as discretizations of $f$ and derive the desired estimates. The central property of local strong monotonicity, implying all this features, will be shown to hold in two situations, giving different characterizations of the "regime of weak corellation" in which CC theory works well: In one proof (Thm. 3.4), we will assume

(I) that the lowest eigenvalue $E^{*}$ of $H$ is simple and isolated from the rest of the spectrum by a non-zero gap $\gamma^{*}$, and

(II) the existence of a sufficiently accurate reference determinant $\Psi_{0}$, constructed from an $N$-dimensional reference subspace $R$ of the one-electron space $H^{1}\left(\mathbb{R}^{3} \times\left\{ \pm \frac{1}{2}\right\}\right)$.

Assumption I excludes the case of system degeneracy in which Coupled Cluster cannot be guaranteed to work well as detailed above. It corresponds to a commom assumption under which results for linear operator eigenvalue problems in the vein of (1.4) may be derived quite straightforwardly, and we will use this feature to derive analogous results for the more complicated CC methods in this work by interpreting the CC function as a perturbation of the operator $H-E^{*}$. Assumption II exempts the analysis from the case of static correlation discussed above, and excluding this situation is indeed a basic condition for the practical applicability of singlereference methods as standard CI or CC for computing the electronic wavefunction (see, e.g., [48], Sect. 1.6).

Our second proof for strong monotonicity (Thm. 3.7) circumvents the assumption on the simple eigenvalue of $H$ and instead assumes that the similarity transformed Hamiltonian $e^{-T(t)} H \mathrm{e}^{T(t)}$ can be decomposed to a sum of two operators $\mathrm{e}^{-T(t)} H \mathrm{e}^{T(t)}=\hat{F}(t)+\hat{U}(t)$, where $\hat{F}:=\left(F-\Lambda_{0}\right) T(t), F-\Lambda_{0}$ being the shifted many-particle Fock operator of the system from unrestricted Hartree-Fock theory [7], and where $\hat{U}(t)$ is a nonlinear function with small Lipschitz constant, correponding to the fluctuation potential of $H$. This corresponds to imposing that the Hamiltonian can be well approximated by a Fock-like operator, so that also this characterization of strong monotonicity stresses the perturbational character of Coupled Cluster theory as outlined above.

The rest of the paper is organized as follows: In Section 2, we will mainly review the content of the previous [79] as far as it is relevant for this work: The reformulation of the electronic Schrödinger equation (1.1) as a root equation for the continuous CC equations (1.8) will be introduced at more length, and some important supplementary results will be compiled. In Section 3, the analytical properties of the CC function as operator between the coefficient space $\mathbb{V}$ and its dual are analyzed; the main result the aforementioned strong monotonicity on a neighbourhood of the solution (Thm. 3.4). The existence and uniqueness statements and quasi-optimality results for the CC method discussed above are then strictly derived from this in Section 4, see Theorems 4.1 and 4.5. Some concluding remarks are given in Section 5. The appended Section 5 discusses some aspects concerned with the practical treatment of the CC equations (1.6) from the viewpoint given in this work.

\section{Preliminaries: From the Schrödinger equation to CC.}

In this section, we fix the mathematical framework needed for the due analysis: In part (i), we introduce our basic notation for the Schrödinger equation (1.1). Parts (ii) and (iii) give an overview of the transition to the continuous Coupled Cluster formulation for this equation, mainly taken from our previous work [79] (with a "continuous CI method" an intermediate stage). Part (iv) contains some important supplementary results, partly taken from [79], that will also be needed later.

(i) Electronic Schrödinger equation. The goal of electronic structure calculations is solution of the $N$-particle electronic Schrödinger equation. It can be stated as a weak operator eigenvalue equation on a suitable solution space $\mathbb{H}^{1}$, which can be defined either as the space

$$
\mathbb{L}^{2} \cap H^{1}\left(\left(\mathbb{R}^{3} \times\left\{ \pm \frac{1}{2}\right\}\right)^{N}\right),
$$


where $\mathbb{L}^{2}$ denotes the antisymmetric functions from $L^{2}\left(\left(\mathbb{R}^{3} \times\left\{ \pm \frac{1}{2}\right\}\right)^{N}\right)$ and $H^{1}(\Omega)$ denotes the Sobolev space of one time weakly differentiable functions over a given measure space $\Omega$ (see, e.g., [80]), or alternatively as a subspace of (2.1) that is an eigenspace of an operator commuting with $H$. The latter results in a restricted, often computationally less demanding formulation on this subspace; in practice, the use of a fixed eigenspace of the $z$-spin operator defined on (2.1) is rather common. On such a fixed space $\mathbb{H}^{1}$, we will use the $\mathbb{L}^{2}$-inner product and the induced norm, denoted by $\langle\cdot, \cdot\rangle$ and $|\cdot|$, respectively, as well as the canonical Sobolev $\mathbb{H}^{1}$-inner product

$$
\langle\Psi, \Phi\rangle_{\mathbb{H}^{1}}:=\langle\Psi, \Phi\rangle+\langle\nabla \Psi, \nabla \Phi\rangle
$$

and the induced Sobolev norm $\|\cdot\|$, given by $\|\Psi\|=\langle\Psi, \Psi\rangle_{\mathbb{H}^{1}}^{\frac{1}{2}}$. In $\mathbb{H}^{1}$, we wish to solve the weak eigenvalue problem

$$
\left\langle\left(H-E^{*}\right) \underline{\Psi}, \Psi\right\rangle=0 \quad \text { for all } \quad \Psi \in \mathbb{H}^{1}
$$

for the lowest eigenvalue $E^{*}$ of the $N$-electron Hamiltonian $H: \mathbb{H}^{1} \rightarrow \mathbb{H}^{-1}$,

$$
H:=-\frac{1}{2} \sum_{i=1}^{N} \Delta_{i}+\sum_{i=1}^{N} \sum_{\substack{j=1 \\ j \neq i}}^{N} \frac{1}{\left|x_{i}-x_{j}\right|}-\sum_{i=1}^{N} \sum_{k=1}^{M} \frac{Z_{k}}{\left|x_{i}-R_{k}\right|} .
$$

In this, the Laplacian $\Delta_{i}$ acts on the $i$ th spatial component $x_{i} \in \mathbb{R}^{3}$ of a wave function $\Psi=$ $\Psi\left(\left(x_{1}, s_{1}\right), \ldots,\left(x_{n}, s_{n}\right)\right)$, and the constants $Z_{k} \in \mathbb{N}, R_{k} \in \mathbb{R}^{3}$ are the charges and positions of the $M$ fixed nuclei. $H$ is bounded and fulfils a Gårding inequality [87] on $\mathbb{H}^{1}$ [88]: There holds

$$
c\|\Psi\|^{2}-\mu\langle\Psi, \Psi\rangle \leq\langle H \Psi, \Psi\rangle ; \quad\langle H \Psi, \Phi\rangle \leq \Lambda\|\Psi\|\|\Phi\|
$$

for all $\Psi, \Phi \in \mathbb{H}^{1}$ and constants $\mu \in \mathbb{R}, c, \Lambda>0$. In the formulation of (2.3), the existence of a smallest eigenvalue is supposed, i.e. the number

$$
\mathcal{E}^{*}:=\inf \left\{\langle H \Psi, \Psi\rangle \mid \Psi \in \mathbb{H}^{1}, \quad\|\Psi\|=1\right\}
$$

has to fulfil

$$
\mathcal{E}^{*}<\inf \operatorname{spec}_{\mathrm{ess}}(H) \text {. }
$$

The bottom inf specess $_{\text {ef }}$ the essential spectrum can be identified with a formalization of the ionization threshold energy of the molecule (the famous HVZ Theorem named after Zhislin, van Winter and Hunziker, see, e.g., $[2,71,88]$ ). The inequality (i) of the above assumption therefore follows [88] if we can assume that for the given configuration of nuclei (as expressed in $H$ ), it is energetically more advantageous for the electrons to stay in the vicinity of the nuclei than to fade away at infinity - which is physically reasonable if we want to compute stable molecules. The validity of (i) can be formally proven for some cases, e.g. for one-atomic molecules or if the number $N$ of electrons is less or equal the total nuclear charge $\sum_{k=1}^{M} Z_{k}$ ([90], confer also the quite exhaustive review [46]). We will in the following assume that $(2.7)$ is valid. This also implies that $\mathcal{E}^{*}$ is the lowest eigenvalue of $H$, i.e. $\mathcal{E}^{*}=E^{*}$, see, e.g., [88].

(ii) The complete CI equations. For reformulation of the eigenvalue equation (2.3) in terms of the Coupled Cluster equations, a basis $\mathbb{B}$ of the space $\mathbb{H}^{1}$ now has to be fixed. We construct $\mathbb{B}$ first by fixing a complete one-particle basis

$$
B:=\left\{\chi_{P} \mid P \in \mathbb{N}\right\}
$$


of the one-electron state space

$$
H^{1}:=H^{1}\left(\mathbb{R}^{3} \times\left\{ \pm \frac{1}{2}\right\}\right) .
$$

We define Slater determinants (as formed from $N$ one-electron functions from $B$ ) by

$$
\Psi_{\left(P_{1}, \ldots, P_{N}\right)}:=\bigwedge_{i=1}^{N} \chi_{P_{i}}:=\frac{1}{\sqrt{N !}} \sum_{\pi \in S(N)}(-1)^{\operatorname{sgn}(\pi)} \chi_{\pi\left(P_{1}\right)} \otimes \ldots \otimes \chi_{\pi\left(P_{N}\right)},
$$

with the sum running over the permutational group $S(N)$ on $N$ elements operating on the indices of $\Psi$ and with $P_{1}, \ldots, P_{N} \in \mathbb{N}$. For $\mathbb{H}^{1}$ given by (2.1), a Slater basis of $\mathbb{L}^{2}$ and $\mathbb{H}^{1}$ is then obtained by letting

$$
\mathbb{B}:=\left\{\Psi_{\mu} \mid \mu \in \mathcal{M}_{0}\right\}, \quad \mathcal{M}_{0}=\left\{\left(P_{1}, \ldots, P_{N}\right) \mid P_{i} \in \mathbb{N}, \quad P_{1}<\ldots<P_{N}\right\} .
$$

If $\mathbb{H}^{1}$ is given by a subspace of (2.1), corresponding to an eigenspace of the $z$-spin operator, $B$ may be chosen as an eigenbasis of the one-electron $z$-spin operator $S_{z} ;(2.9)$ then is an eigenbasis of $S_{z}$ and can be restricted accordingly (see [78] for an explicit construction). To obtain a well-defined infinite-dimensional Coupled Cluster theory as developed in [79] and reviewed below, one has to assume that the reference determinant

$$
\Psi_{0}:=\bigwedge_{i=1}^{N} \chi_{i}
$$

as canonically formed from the set of the first $N$ basis functions

$$
B_{\mathrm{occ}}:=\left\{\chi_{1}, \ldots, \chi_{N}\right\}
$$

from $B$, is non-orthogonal to the sought solution $\underline{\Psi}$,

$$
\left\langle\underline{\Psi}, \Psi_{0}\right\rangle \neq 0
$$

A reference $\Psi_{0}$ fixed, equation (2.3) can be reformulated in terms of the Configuration Interaction ansatz: "Find $\underline{\Psi}=\Psi_{0}+\Psi^{*} \in \mathbb{H}^{1}$ with $\Psi^{*}$ orthogonal to $\Psi_{0}$ such that

$$
\left\langle H\left(\Psi_{0}+\Psi^{*}\right), \Psi_{\mu}\right\rangle=E\left\langle\Psi_{0}+\Psi^{*}, \Psi_{\mu}\right\rangle \text { for all } \Psi_{\mu} \in \mathbb{B}, \quad \text { where } \Psi^{*} \perp \Psi_{0} . "
$$

Note that the orthogonality of $\Psi^{*}$ and $\Psi_{0}$ also implies the so-called intermediate normalization condition

$$
\left\langle\underline{\Psi}, \Psi_{0}\right\rangle=1 .
$$

(iii) Excitation and cluster operators; the coefficient space $\mathbb{V}$; the continuous Coupled Cluster equations. Coupled Cluster theory reformulates Equation (2.13) by an exponential ansatz in terms of so-called cluster operators, themselves built of excitation operators to be introduced in this section. By defining a suitable coefficient space $\mathbb{V}$ reflecting the set of admissible corrections $\Psi^{*} \in \mathbb{H}^{1}$, we may then formulate the continuous CC equations.

To keep the exposition as compact as possible, we decided to give a slightly informal definition of the cluster operators sufficient for the later analysis in this work. A formal definition in terms of creation and annihilation operators is included in the Appendix, see Section (ii). Following conventions of quantum chemistry literature, we will call functions $\chi_{I} \in B_{\text {occ }}$ from (2.11) occupied orbitals and index them by letters $I, J, K, \ldots \in$ occ; functions $\chi_{A} \in B \backslash B_{\text {occ }}$ are called virtual orbitals, denoted by $A, B, C, \ldots \in$ virt. Undesignated indices are denoted as $P, Q, R$, etc. Let $\mathcal{M}=\mathcal{M}_{0} \backslash\left\{\mu_{0}\right\}$, where $\mu_{0}=(1, \ldots, N)$ is the index belonging to the reference $\Psi_{0}$. Then each multi-index $\mu \in \mathcal{M}$ can formally be obtained by exchanging in $\mu_{0}$ certain indices $\left\{I_{1}, \ldots, I_{r}\right\} \subseteq$ occ 
(namely those not contained in $\mu$ ) against the virtual orbitals $\left\{A_{1}, \ldots, A_{r}\right\} \subseteq$ virt contained in $\mu$. This idea is used to define for each $\mu \in \mathcal{M}$ an excitation operator

$$
X_{\mu}:=X_{I_{1}, \ldots, I_{r}}^{A_{1}, \ldots, A_{r}}
$$

where $\left\{I_{1}, \ldots, I_{r}\right\}$ are the occupied orbitals not contained in $\mu$ and $\left\{A_{1}, \ldots, A_{r}\right\}$ are the virtuals contained in $\mu$, each in increasing order. The action of $X_{\mu}$ can be characterized by its effect on basis functions $\Psi_{\nu}=$ $\Psi_{\left(P_{1}, \ldots, P_{N}\right)} \in \mathbb{B}$ : If $\left\{P_{1}, \ldots, P_{N}\right\}$ contains all indices in $\left\{I_{1}, \ldots, I_{r}\right\}$ and none of the set $\left\{A_{1}, \ldots, A_{r}\right\}$, the action of $X_{\mu}$ gives another Slater determinant $\Psi_{\alpha}$ of index $\alpha=\alpha(\mu, \nu) \in \mathcal{M}$, equipped with a uniquely determined sign (see [79]): We have

$$
X_{\mu} \Psi_{\nu}= \pm \Psi_{\alpha(\mu, \nu)},
$$

with $\alpha(\mu, \nu)$ obtained by replacing in $\nu$ the occupied indices $I_{1}, \ldots, I_{r}$ by the virtual orbitals $A_{1}, \ldots, A_{r}$. Otherwise, i.e., if one of the occupied indices $\left\{I_{1}, \ldots, I_{r}\right\}$ is not contained in $\left\{P_{1}, \ldots, P_{N}\right\}$ or one of the virtual indices $\left\{A_{1}, \ldots, A_{r}\right\}$ is contained in $\left\{P_{1}, \ldots, P_{N}\right\}$, we let $X_{\mu} \Psi_{\nu}=0$. By linear continuation, we obtain a continuous linear operator

$$
X_{\mu}: \mathbb{L}^{2} \rightarrow \mathbb{L}^{2},
$$

called the excitation operator belonging to the index $\mu$. Note that by definition, two essential prerequisites for the exponential ansatz in CC hold: We have

$$
X_{\mu} X_{\nu}=X_{\nu} X_{\mu}, \quad \text { and } \quad X_{\mu} \Psi_{0}= \pm \Psi_{\mu}
$$

for all $\mu, \nu \in \mathcal{M}$. Now, every function $\Psi=\Psi_{0}+\Psi^{*}=\Psi_{0}+\sum_{\mu \in \mathcal{M}} \hat{t}_{\mu} \Psi_{\mu} \in \mathbb{L}^{2}$ can be expressed via excitation operators acting on the reference determinant:

$$
\Psi=\Psi_{0}+\Psi^{*}=\Psi_{0}+\sum_{\mu \in \mathcal{M}} \hat{t}_{\mu} \Psi_{\mu}=\Psi_{0}+\sum_{\mu \in \mathcal{M}} t_{\mu} X_{\mu} \Psi_{0}=:(I+T(t)) \Psi_{0},
$$

where $t_{\mu}= \pm \hat{t}_{\mu}$ with the sign fixed by the second equation in (2.17). The operator

$$
T(t)=\sum_{\mu \in \mathcal{M}} t_{\mu} X_{\mu}
$$

is called the cluster operator belonging to the correction $\Psi^{*}$, respectively, the corresponding coefficient vector $\left(t_{\mu}\right)_{\mu \in \mathcal{M}}$.

By the Schrödinger equation (2.3), respectively its CI form (2.13), we are looking for a correction $\Psi^{*}=$ $\sum_{\mu \in \mathcal{M}} t_{\mu} \Psi_{\mu}$ contained in the energy space $\mathbb{H}^{1}$, and the set of all functions (2.18) has to be restricted accordingly. In terms of the corresponding vector $t=\left(t_{\mu}\right)_{\mu \in \mathcal{M}}$, this restriction can be formulated by defining a subspace $\mathbb{V} \subseteq \ell_{2}(\mathcal{M})$ by

$$
\mathbb{V}:=\left\{t \in \ell_{2}(\mathcal{M}) \mid\|t\|_{\mathbb{V}}<\infty\right\} .
$$

where, with the Sobolev inner product (2.2),

$$
\langle t, s\rangle_{\mathbb{V}}:=\left\langle\sum_{\mu \in \mathcal{M}} t_{\mu} \Psi_{\mu}, \sum_{\nu \in \mathcal{M}} s_{\nu} \Psi_{\nu}\right\rangle_{\mathbb{H}^{1}}, \quad\|t\|_{\mathbb{V}}:=\langle t, t\rangle_{\mathbb{V}}^{1 / 2} .
$$

$\mathbb{V}$ is a Hilbert space, and it holds $t \in \mathbb{V}$ iff $\Psi^{*}=\sum_{\mu \in \mathcal{M}} t_{\mu} \Psi_{\mu} \in \mathbb{H}^{1}$. Using this definition, we can bring the canonical (finite-dimensional) CC equations (1.6) together with the context of functional analysis. The main result enabling this step is the following [79], Theorem 4.1. 
Theorem 2.1 ( $\mathbb{H}^{1}$-continuity of cluster operators).

(i) For $\Psi^{*}=\sum_{\mu \in \mathcal{M}} t_{\mu} \Psi_{\mu} \in$ span $\mathbb{B}$ and $T=T(t)$ the associated cluster operator defined by (2.19), we have

$$
\Psi^{*} \in \mathbb{H}^{1} \Leftrightarrow T \in B\left(\mathbb{H}^{1}, \mathbb{H}^{1}\right),
$$

i.e., $T$ is bounded as mapping $\mathbb{H}^{1} \rightarrow \mathbb{H}^{1}$. Moreover, with constants depending only on the number $N$ of electrons and on $\left\|\Psi_{0}\right\|$,

$$
\|T\|_{\mathbb{H}^{1} \rightarrow \mathbb{H}^{1}} \sim\left\|\Psi^{*}\right\|=\|t\|_{\mathbb{V}} .
$$

(ii) For the $\mathbb{L}^{2}$-adjoint $T^{\dagger}$ of any cluster operator $T \in B\left(\mathbb{L}^{2}, \mathbb{L}^{2}\right)$, there holds

$$
T^{\dagger} \in B\left(\mathbb{H}^{1}, \mathbb{H}^{1}\right), \quad\left\|T^{\dagger}\right\|_{\mathbb{H}^{1} \rightarrow \mathbb{H}^{1}} \lesssim\left\|\Psi^{*}\right\|=\|t\|_{\mathbb{V}} .
$$

where again, the constant depends only on the number $N$ of electrons and on $\left\|\Psi_{0}\right\|$.

From this result, the electronic Schrödinger equation can be reformulated as a root equation for the so-called Coupled Cluster function ${ }^{3}$, see [79], Theorems 5.3, 5.5.

Theorem 2.2. Let $\underline{\Psi} \in \mathbb{H}^{1}$ with $\left\langle\Psi_{0}, \underline{\Psi}\right\rangle \neq 0$ and $E \in \mathbb{R}$. The following are equivalent:

(i) $\underline{\Psi}$ solves the (weak, CI) eigenproblem

$$
\langle\Phi,(H-E) \underline{\Psi}\rangle=0, \quad \text { for all } \Phi \in \mathbb{H}^{1} .
$$

(ii) $\underline{\Psi}=\mathrm{e}^{T} \Psi_{0}$ for some cluster operator $T \in B\left(\mathbb{H}^{1}, \mathbb{H}^{1}\right)$ which fulfils the (continuous) linked Coupled Cluster equations,

$$
E=\left\langle\Psi_{0}, H \mathrm{e}^{T} \Psi_{0}\right\rangle, \quad\left\langle\Psi_{\mu}, \mathrm{e}^{-T} H \mathrm{e}^{T} \Psi_{0}\right\rangle=0 \quad \text { for all } \mu \in \mathcal{M} .
$$

(iii) There is a coefficient vector $t^{*}=\left(t_{\mu}^{*}\right)_{\mu \mathcal{M}} \in \mathbb{V}$ such that $T=T\left(t^{*}\right)$ and that $t^{*}$ is a root of the continuous Coupled Cluster function

$$
f: \mathbb{V} \rightarrow \mathbb{V}^{\prime}, \quad t \mapsto\left(\left\langle\Psi_{\mu}, \mathrm{e}^{-T(t)} H \mathrm{e}^{T(t)} \Psi_{0}\right\rangle\right)_{\mu \in \mathcal{M}},
$$

that is, $t^{*}$ solves the nonlinear continuous CC equation

$$
f\left(t^{*}\right)=0 \in \mathbb{V}^{\prime}
$$

For the proof of the fact that $f$ maps $\mathbb{V} \rightarrow \mathbb{V}^{\prime}$ continuously, which is not given in [79], cf. Lemma 3.1.

The canonical, finite-dimensional CC equations, derived in the introduction as an approximation to a finitedimensional Galerkin ("full-CI") limit, can now also be viewed as a Galerkin approximation for the nonlinear continuous Coupled Cluster function $f$ [74]: Let $\mathcal{M}_{d} \subset \mathcal{M}$, so that $\mathbb{B}_{d}=\left\{\Psi_{0}\right\} \cup\left\{\Psi_{\mu} \mid \mu \in \mathcal{M}_{d}\right\} \subseteq \mathbb{B}$ is a selection of basis functions containing $\Psi_{0}$, and denote the corresponding coefficient space by $\mathbb{V}_{d} \subseteq \mathbb{V}$. The Galerkin projection of the continuous CC equation (2.28), i.e., the set of equations

$$
\left\langle s_{d}, f\left(t_{d}^{*}\right)\right\rangle=0 \quad \text { for all } s_{d} \in \mathbb{V}_{d}, \quad E_{d}=\left\langle\Psi_{0}, H \mathrm{e}^{T\left(t_{d}\right)} \Psi_{0}\right\rangle
$$

\footnotetext{
${ }^{3}$ The Coupled Cluster function is defined on the infinite dimensional space $\mathbb{V}$, and some readers might therefore prefer the term "(nonlinear) operator" and denote it by a capital letter. To keep consistent with quantum chemistry literature,we will stick to the physicist's/chemist's nomenclature of "the Coupled Cluster function" $f$ here.
} 
to be solved for $t_{d} \in \mathbb{V}_{d}$, then corresponds to the classical, finite-dimensional CC equations, based on the reference determinant $\Psi_{0}$ and the trial space $\mathbb{V}_{d}=$ span $\mathbb{B}_{d}$. In terms of wave functions, this corresponds to a discretisation of the linked CC equations

$$
\left\langle\Psi_{\mu}, \mathrm{e}^{-T\left(t_{d}\right)} H \mathrm{e}^{T\left(t_{d}\right)} \Psi_{0}\right\rangle=0 \quad \text { for all } \mu \in \mathcal{M}_{d}, \quad E_{d}=\left\langle\Psi_{0}, H \mathrm{e}^{T\left(t_{d}\right)} \Psi_{0}\right\rangle
$$

The enabling of this viewpoint is the main achievement of [79], and we will exploit it strongly in the due analysis in this work.

(iv) Supplementary results. Two more technical results will be needed in this work, and we compile them here to refer to them later.

Lemma 2.3 (The algebra of $\mathbb{H}^{1}$-continuous cluster operators, [79], Lem. 5.2).

Between the set of cluster operators belonging to corrections $\Psi^{*} \in \mathbb{H}^{1}$ (cf. Thm. 2.1),

$$
\mathcal{T}=\{T(t) \mid t \in \mathbb{V}\}=\left\{T \mid T \in B\left(\mathbb{H}^{1}, \mathbb{H}^{1}\right) \text { is a cluster operator }\right\}
$$

and the set

$$
I+\mathcal{T}=\{I+T \mid T \in \mathcal{T}\} \subseteq B\left(\mathbb{H}^{1}, \mathbb{H}^{1}\right),
$$

the exponential function ${ }^{4} \exp (T)=\sum_{i=0}^{N} T^{i} / i$ ! establishes a $C^{\infty}$, locally Lipschitz continuous bijection. This also holds if in the above, $\mathbb{V}$ is replaced by a subspace $\mathbb{V}_{d} \subseteq \mathbb{V}$.

The second result in this section shows that from (2.5), it follows that $H-E_{0}$ is a bounded, $\mathbb{H}^{1}$-elliptic mapping on the orthogonal complement of the eigenspace belonging to $E_{0}$. This fact lays the basis for the monotonicity of the Coupled Cluster function (Thm. 3.4), on which in turn the whole error analysis given here will be based. We give a more general result in terms of a Gelfand triple $V \hookrightarrow X \hookrightarrow V^{\prime}$, i.e. $V \subseteq X$ are Hilbert spaces with $V$ dense in $X$ and the embedding $V \hookrightarrow X$ continuous (see, e.g., [40]). The situation we are aiming at here is $V=\mathbb{H}^{1}, X=\mathbb{L}^{2}$.

Lemma 2.4 (Ellipticity results for $H$ ).

(i) Let $V \hookrightarrow X \hookrightarrow V^{\prime}$ be a Gelfand triple, and and let $A: V \rightarrow V^{\prime}$ be a symmetric operator which is bounded from below by a Gårding estimate

$$
\langle A v, v\rangle \geq c\|v\|_{V}^{2}-\mu\|v\|_{X}^{2}
$$

with constants $c>0, \mu \in \mathbb{R}$. If additionally, $A$ is $X$-elliptic, i.e.

$$
\langle A v, v\rangle \geq \gamma^{*}\|v\|_{X}^{2} \quad \text { for all } \quad v \in V
$$

for some $\gamma^{*}>0$, then $A$ is also $V$-elliptic, i.e. for some $\gamma^{\prime}>0$,

$$
\langle A v, v\rangle \geq \gamma^{\prime}\|v\|_{V}^{2} \text { for all } v \in V .
$$

(ii) If (2.32) only holds on a closed subspace $U \subseteq V$, this still implies

$$
\langle A u, u\rangle \geq \gamma^{\prime}\|u\|_{V}^{2} \text { for all } u \in U .
$$

Proof. To prove (i), we let $q:=\gamma^{*} /\left(\mu+\gamma^{*}\right)<1$ and use that $\langle A v, v\rangle$ can be expressed as

$$
q\langle A v, v\rangle+(1-q)\langle A v, v\rangle \geq q c\|v\|_{V}^{2}+(\gamma-q(\mu+\gamma))\|v\|_{X}^{2}=q c\|v\|_{V}^{2} .
$$

To prove statement (ii), we denote the projector on $U$ by $P$; the restriction $\tilde{A}=P A P$ is symmetric and fulfils the conditions $(2.31),(2.32)$ with $A$ replaced by $\tilde{A}, V$ replaced by $U$. Part (i) thus implies

$$
\langle\tilde{A} u, u\rangle \geq \gamma^{\prime}\|u\|_{V}^{2} \quad \text { for all } \quad u \in U
$$

which gives (2.34).

\footnotetext{
${ }^{4}$ Note that the exponential series terminates at $N$ as a consequence to the nilpotency property $X_{\mu}^{2}=0$ of cluster operators.
} 


\section{Analytical properties of the coupled Cluster function}

In this section, we give two characterizations of situations in which the Coupled Cluster function $f$ has the property of local strong monotonicity. This property will be the main tool to obtain the estimates for the CC method given in Section 4. In (i), we derive some preliminary results and definitions. In (ii), the first characterization is given (Thm. 3.4) and the assumptions made to obtain the property are being dicussed; it is proved in (iii). The second characterization is obtained in (iv), Theorem 3.7.

(i) Preliminary statements on the CC function. We start by the next lemma compiling some general properties of $f$, which were already shown in [82] for the discrete case.

Lemma 3.1 (The Coupled Cluster function).

The Coupled Cluster function (2.27) is a $C^{\infty}$-mapping from $\mathbb{V}$ to its dual $\mathbb{V}^{\prime} . f$ and all derivatives $f^{(n)}$ of $f$ are Lipschitz-continuous on bounded domains of $\mathbb{V}$.

Proof. Let us denote by $\langle\cdot, \cdot\rangle_{\ell_{2}}$ the usual $\ell_{2}(\mathcal{M})$-inner product. Then, for $s, t \in \mathbb{V}$, we obtain with the use of $(2.5)$ and Theorem 2.1 that

$$
\langle f(t), s\rangle_{\ell_{2}}=\sum_{\mu \in \mathcal{M}}\left\langle s_{\mu} \Psi_{\mu}, \mathrm{e}^{-T(t)} H \mathrm{e}^{T(t)} \Psi_{0}\right\rangle \leq\left\|T(s) \Psi_{0}\right\|\left\|\mathrm{e}^{-T(t)} H \mathrm{e}^{T(t)} \Psi_{0}\right\|_{\mathbb{H}^{-1}} \leq C\|s\|_{\mathbb{V}},
$$

where the constant $C$ depends on $\|t\|_{\mathbb{V}}, N$ and on $\left\|\Psi_{0}\right\| \leq\|\underline{\Psi}\|+\varepsilon \cdot\langle f(t), \cdot\rangle_{\ell_{2}}$ thus defines a continuous functional on $\mathbb{V}$. In particular, the Lipschitz continuity of $f$ on bounded domains follows from a short calculation based on the same property of $T \mapsto \mathrm{e}^{T}$ as mapping $\mathcal{T} \rightarrow I+\mathcal{T}$, see Lemma 2.3. $f$ is $C^{\infty}$ as a composition of $C^{\infty}$-functions. For the proof that all derivatives of $f$ are Lipschitz-continuous on bounded domains, see [82] which transfers to our case.

The important property of local strong monotonicity, being the main property used in the proof of the error estimates and optimality results in Section 4, is defined as follows.

Definition 3.2 (Local strong monotonicity).

A function $f: \mathbb{V} \rightarrow \mathbb{V}^{\prime}$ is called locally strongly monotone at $t^{*}=\left(t_{\mu}^{*}\right)_{\mu \in \mathcal{M}}$, if there are constants $\gamma, \delta>0$ such that

$$
\langle f(s)-f(t), s-t\rangle \geq \gamma \cdot\|s-t\|_{\mathbb{V}}^{2}
$$

holds for all $s, t \in B_{\delta}\left(t^{*}\right):=\left\{v \in \mathbb{V} \mid\left\|t^{*}-v\right\|_{\mathbb{V}}<\delta\right\}$.

We note a consequence of strong monotonicity that will be of use later:

Corollary 3.3 (Properties of the derivative of $f$ ).

Let $f$ be locally strongly monotone at $t^{*}$. For $s \in B_{\delta}\left(t^{*}\right)$, the derivatives $D f(s) \in L\left(\mathbb{V}, \mathbb{V}^{\prime}\right)$ of the Coupled Cluster function $f$ at $s$ are uniformly bounded, $\mathbb{V}$-coercive linear operators, i.e. there is a $C>0$ such that

$$
\langle D f(s) u, v\rangle \leq C \cdot\|u\|_{\mathbb{V}}\|v\|_{\mathbb{V}}, \quad\langle D f(s) u, u\rangle \geq \gamma\|u\|_{\mathbb{V}}^{2}
$$

holds for all $s \in B_{\delta}\left(t^{*}\right), u, v \in \mathbb{V}$ (with $\gamma$ from (3.1)).

Proof. The CC function $f$ is $C^{\infty}$ by Lemma 3.1, and it was already noted above that $D f(t)$ is locally Lipschitz continuous, implying the uniform boundedness. For the coercivity, we expand $f$ into a Taylor series, $f\left(s+u^{\prime}\right)-$ $f(s)=D f(s) u^{\prime}+\mathcal{O}\left(\left\|u^{\prime}\right\|_{\mathbb{V}}^{2}\right)$. Inserting this into the strong monotonicity estimate (3.1), one obtains by choosing $u^{\prime}=u / c$ small enough and then using linearity that

$$
\langle D f(s) u, u\rangle \geq \gamma\|u\|_{\mathbb{V}}^{2}-\mathcal{O}\left(\|u\|_{\mathbb{V}}^{3}\right) \geq \gamma\|u\|_{\mathbb{V}}^{2}-\varepsilon
$$

holds for all $u \in \mathbb{V}$ and $\varepsilon>0$. This completes the proof. 
(ii) Strong monotonicity of $f$ (first characterization). To obtain our first monontonicity statement for $f$, we will use the formal counterpart of two practical working assumptions of CC theory as exposed in the introduction, i.e., we will exclude static correlation and system degeneracy. The two mathematical assumptions corresponding to this assumptions will now be introduced and discussed in more detail below. They read as follows:

Assumption AI. The lowest eigenvalue of $E^{*}$ of $H$ is simple.

Assumption AII. The reference determinant (2.10) formed from $B_{\text {occ }}$ approximates the full solution $\underline{\Psi}$ to a certain extent, so that for $\Psi^{*}=\Psi_{0}-\underline{\Psi}$,

$$
\left\|\Psi^{*}\right\|<\varepsilon
$$

with $\varepsilon$ specified later.

Let us comment on these assumptions in detail:

Assumption AI: Although assumtions as the above are rather common in eigenvalue theory, results in which cases it holds for the Hamiltonian are rather scarce to our best knowledge. It can be shown that for some fixed numbers of electrons $(N=2,4,10)$ in the field of a single atomic nucleus of sufficiently large charge, the ground state is simple, while it is not for $N=1,3,5,6,7,8,9[36]$. What is more, even the hydrogen ground state possesses two identical solutions of spin up and spin down, respectively, if the full space (2.1) is considered. Note though that in this case, restriction to eigenspaces of the $z$-spin operator yields two identical problems with simple lowest eigenvalues, and a similar reduction of dimensions can be observed in the other cases if the spin is restricted, see Table 2 in [36]. We therefore stress that the whole analysis in this paper also holds for $\mathrm{CC}$ equations formulated on subspaces of fixed $z$-spin and choices of subspaces exploiting, for instance, spacial symmetries of simple molecules, and that these restricted calculations are actually what is almost exclusively computed in practice; for more complicated molecules, on the other hand, spatial symmetries are less probable to occur.

Assumption I provides (together with Assumption II below) a sufficient condition for CC to behave well, and on the other hand, there are well-known examples with non-unique ground states or small gaps where the efficiency of CC deteriorates, famous test cases being, e.g., the dissociation of $N_{2}[57,63]$ or the calculation of metals or transition metals, for which even multi-reference approaches may fail $[8,76]$.

Assumption AII: The second assumption reflects the fact that Coupled Cluster is a perturbational ansatz which works well if the quality of the reference determinant is sufficiently good and thus corresponds to a basic working assumption in practical Coupled Cluster calculations: In quantum chemist's language, if Assumption 3 holds, the solution $\underline{\Psi}$ is said to be subject to dynamic corellation, as opposed to static correlation, describing the situation where only two or more determinants give sufficient results [48] as in the case of, e.g., ozone. In these cases, a method based on more than one determinant will have to be utilized, as for instance the technically rather complicated multi-reference-CC ansätze $[15,66,68,72]$, or calculations will have to include a large number of basis functions from $\mathbb{B}$. Related practical heuristics indicating a posteriori the quality of a $\mathrm{CC}$ calculation are that of the $T 1$ or $D 1$ diagnostics [54,56], in which the size of the particular coefficients in the CC amplitude vector $t_{d}$ are considered as a measure for static correlation.

Based on these assumptions, we now give the announced first characterization for strong monotonicity.

Theorem 3.4 (Local strong monotonicity of the CC function (1)).

Let $f$ denote a CC function based on a Hamiltonian $H$ fulfilling Assumption AI and a reference $\Psi_{0}$ fulfilling Assumption AII. The Coupled Cluster function $f$ then is strongly monotone in a neighbourhood of its solution $t^{*}=\left(t_{\mu}^{*}\right)_{\mu \in \mathcal{M}}$, i.e., (3.1) holds for all $s, t \in B_{\delta}\left(t^{*}\right):=\left\{v \in \mathbb{V} \mid\left\|t^{*}-v\right\|_{\mathbb{V}}<\delta\right\}$. H fixed, the constants are uniform for all Coupled Cluster methods that fulfil the estimate (3.3) uniformly. 
(iii) Proof of Theorem 3.4. We start by proving an auxiliary lemma based on Lemma 2.4. It is the core ingredient to the Proof of Theorem 3.4 performed thereafter.

Lemma 3.5. Let $U_{0}:=\operatorname{span}\left\{\Psi_{0}\right\}$. If the reference determinant $\Psi_{0}$ lies sufficiently close to the (intermediately normed) solution $\underline{\Psi}$ of the Schrödinger equation and $E^{*}$ is a simple eigenvalue of $H$, the restriction of the mapping $H-E^{*}$ to the orthogonal complement $U_{0}^{\perp}$ of $U_{0}$ is $\mathbb{H}^{1}$-elliptic, i.e.

$$
\left\langle\Psi,\left(H-E^{*}\right) \Psi\right\rangle \geq \gamma^{\prime}\|\Psi\|^{2}
$$

holds for some $\gamma^{\prime}>0$ and all $\Psi \in U_{0}^{\perp}$.

Proof. We show that $H-E^{*}$ is elliptic on $U_{0}^{\perp}$ with respect to the $\mathbb{L}^{2}$-inner product $\langle\cdot, \cdot \cdot\rangle$ and then apply Lemma 2.4(ii). We let $\underline{P}, P_{0}$ be the $\mathbb{L}^{2}$-orthogonal projectors on $\operatorname{span}\{\underline{\Psi}\}, \operatorname{span}\left\{\Psi_{0}\right\}$, respectively, and denote the spectral gap by $\gamma^{*}:=\inf \left(\operatorname{spec}(H) \backslash\left\{E^{*}\right\}\right)-E^{*}$ and, as above, the $\mathbb{L}^{2}$-norm by $|\cdot|$. We have $H-E^{*}=0$ on $\operatorname{span}\{\underline{\Psi}\}$, thus, because $\underline{P}$ projects on an eigenspace of $H$ and thus commutes with $H$, there holds for any $\Psi \in U_{0}^{\perp}$ that

$$
\left\langle\Psi,\left(H-E^{*}\right) \Psi\right\rangle=\left\langle(I-\underline{P}) \Psi,\left(H-E^{*}\right)(I-\underline{P}) \Psi\right\rangle \quad \geq \gamma^{*}|(I-\underline{P}) \Psi|^{2} .
$$

We want to use $\left(I-P_{0}\right) \Psi=\Psi$, and compute the difference of the projectors: Letting $\underline{\Psi}:=\underline{\Psi} /|\underline{\Psi}|$, a short calculation shows that

$$
\left\|P_{0}-\underline{P}\right\|_{\mathbb{L}^{2} \rightarrow \mathbb{L}^{2}}=\max _{f \in \mathbb{L}^{2},|f|=1}\left|\left\langle f, \Psi_{0}\right\rangle \Psi_{0}-\langle f, \underline{\bar{\Psi}}\rangle \underline{\bar{\Psi}}\right| \leq 2\left|\Psi_{0}-\underline{\bar{\Psi}}\right| .
$$

Using orthogonality of $\Psi_{0}$ and $T \Psi_{0}$, there holds with $\tau=\left|T \Psi_{0}\right|,\left|\Psi_{0}\right|=1$ that $\underline{\bar{\Psi}}=\left(\Psi_{0}+T \Psi_{0}\right) /\left(1+\tau^{2}\right)^{1 / 2}$, and one easily sees by orthogonal decomposition that

$$
\left|\Psi_{0}-\underline{\bar{\Psi}}\right|^{2}=\left(1-\frac{1}{\left(1-\tau^{2}\right)^{\frac{1}{2}}}\right)^{2}+\frac{\tau^{2}}{\left(1-\tau^{2}\right)^{\frac{1}{2}}}=2\left(\frac{1}{\left(1+\tau^{2}\right)}-\frac{1}{\left(1+\tau^{2}\right)^{\frac{1}{2}}}\right)=4 \tau^{2}+\mathcal{O}\left(\tau^{4}\right) .
$$

Therefore, we can for instance choose $\tau=\left|\Psi_{0}-\underline{\Psi}\right|$ such that $\left\|P_{0}-\underline{P}\right\|_{\mathbb{L}^{2} \rightarrow \mathbb{L}^{2}} \leq \frac{1}{2}$, and using $\left(I-P_{0}\right) \Psi=\Psi$ there follows

$$
\gamma^{*}|(I-\underline{P}) \Psi|^{2} \geq \gamma^{*}\left(\left|\left(I-P_{0}\right) \Psi\right|-\left|\left(P_{0}-\underline{P}\right) \Psi\right|\right)^{2} \geq \frac{1}{4} \gamma^{*}|\Psi|^{2} .
$$

$H-E^{*}$ is thus $\mathbb{L}^{2}$-elliptic on the complement of $U_{0}$. Therefore - and because the Hamiltonian fulfils Gårding's inequality on $\mathbb{H}^{1}$, see (2.5) - Lemma 2.4 (ii) implies that there is a constant $\gamma^{\prime}$ such that (3.4) holds for all $\Psi \in U_{0}^{\perp}$.

Proof of Theorem 3.4 (Strong monotonicity of $f$ ). To show (3.1), we denote the cluster operator belonging to $t^{*}$ by $T=\sum_{\mu \in \mathcal{M}} t_{\mu}^{*} X_{\mu}$. We let $g_{1}:=s-t^{*}, g_{2}:=t-t^{*}$ and write the corresponding cluster operators as $G_{1}, G_{2}$. We expand $\mathrm{e}^{G_{i}}, \mathrm{e}^{-G_{i}}, i=1,2$, into a series to obtain

$$
\mathrm{e}^{-T-G_{i}} H \mathrm{e}^{T+G_{i}} \Psi_{0}=\mathrm{e}^{-T} H \mathrm{e}^{T} \Psi_{0}-G_{i} \mathrm{e}^{-T} H \mathrm{e}^{T} \Psi_{0}+\mathrm{e}^{-T} H \mathrm{e}^{T} G_{i} \Psi_{0}+\mathcal{O}\left(\left\|g_{i}\right\|_{\mathbb{V}}^{2}\right) .
$$

Thus, with $G=G_{1}-G_{2}$,

$$
\begin{aligned}
\langle f(s)-f(t), s-t\rangle & =\left\langle f\left(t^{*}+g_{1}\right)-f\left(t^{*}+g_{2}\right), g_{1}-g_{2}\right\rangle \\
& :=\left\langle G \Psi_{0}, \mathrm{e}^{-T-G_{1}} H \mathrm{e}^{T+G_{1}} \Psi_{0}\right\rangle-\left\langle G \Psi_{0}, \mathrm{e}^{-T-G_{2}} H \mathrm{e}^{T+G_{2}} \Psi_{0}\right\rangle \\
& \geq\left\langle G \Psi_{0}, \mathrm{e}^{-T} H \mathrm{e}^{T} G \Psi_{0}\right\rangle-\left\langle G^{\dagger} G \Psi_{0}, \mathrm{e}^{-T} H \mathrm{e}^{T} \Psi_{0}\right\rangle-\mathcal{O}\left(\left\|g_{i}\right\|_{\mathbb{V}}^{3}\right)=: \quad(*)
\end{aligned}
$$


by the Cauchy-Schwarz inequality and Theorem 2.1. Using $\mathrm{e}^{-T} H \mathrm{e}^{T} \Psi_{0}=E^{*} \Psi_{0}$, we have

$$
\left\langle G^{\dagger} G \Psi_{0}, \mathrm{e}^{-T} H \mathrm{e}^{T} \Psi_{0}\right\rangle=\left\langle G^{\dagger} G \Psi_{0}, E^{*} \Psi_{0}\right\rangle=E^{*}\left\langle G \Psi_{0}, G \Psi_{0}\right\rangle .
$$

Thus, $(*)$ coincides up to second order with

$$
\left\langle G \Psi_{0}, \mathrm{e}^{-T} H \mathrm{e}^{T} G \Psi_{0}\right\rangle-E^{*}\left\langle G \Psi_{0}, G \Psi_{0}\right\rangle=\left\langle G \Psi_{0}, \mathrm{e}^{-T}\left(H-E^{*}\right) \mathrm{e}^{T} G \Psi_{0}\right\rangle,
$$

and choosing an appropriate $\delta$ and $\left\|g_{1}\right\|_{\mathbb{V}},\left\|g_{2}\right\|_{\mathbb{V}}<\delta$, it suffices to show that this expression is bounded from below by $c \cdot\|g\|_{\mathbb{V}}^{2}$. We expand $\mathrm{e}^{T}, \mathrm{e}^{-T}$ into a power series as above to obtain

$$
\begin{aligned}
& \left\langle G \Psi_{0}, \mathrm{e}^{-T}\left(H-E^{*}\right) \mathrm{e}^{T} G \Psi_{0}\right\rangle \\
= & \left\langle G \Psi_{0},\left(H-E^{*}\right) G \Psi_{0}\right\rangle+\left\langle G \Psi_{0},\left(H-E^{*}\right)\left(T-T^{\dagger}\right) G \Psi_{0}\right\rangle-\mathcal{O}\left(\left\|t^{*}\right\|_{\mathbb{V}}^{2}\|g\|_{\mathbb{V}}^{2}\right) \\
\geq & \gamma^{\prime}\left\|G \Psi_{0}\right\|^{2}-\left(\Lambda-E^{*}\right)\left\|T-T^{\dagger}\right\|_{\mathbb{H}^{1} \rightarrow \mathbb{H}^{1}}\left\|G \Psi_{0}\right\|^{2}-\mathcal{O}\left(\left\|t^{*}\right\|_{\mathbb{V}}^{2}\|g\|_{\mathbb{V}}^{2}\right)
\end{aligned}
$$

where Lemma 3.5 was used in the last step, and the constant $\Lambda$ stems from the upper bound (2.5) for the norm of $H$. Theorem 2.1 states $\|T\|_{\mathbb{H}^{1} \rightarrow \mathbb{H}^{1}},\left\|T^{\dagger}\right\|_{\mathbb{H}^{1} \rightarrow \mathbb{H}^{1}} \lesssim\left\|t^{*}\right\|_{\mathbb{V}} \sim\left\|\Psi^{*}\right\|,\left\|G \Psi_{0}\right\| \gtrsim\|g\|_{\mathbb{V}}$; thus, if $\left\|\Psi^{*}\right\|$ is small enough, this gives constants $c, \gamma^{\prime \prime}, \gamma>0$ such that

$$
\left\langle G \Psi_{0}, \mathrm{e}^{-T}\left(H-E^{*}\right) \mathrm{e}^{T} G \Psi_{0}\right\rangle \geq \gamma^{\prime \prime}\|g\|_{\mathbb{V}}^{2}-c\left\|t^{*}\right\|_{\mathbb{V}}\|g\|_{\mathbb{V}}^{2} \geq \gamma\|g\|_{\mathbb{V}}^{2} .
$$

The proof is now finished by noting that all the respective constants are uniform for all Coupled Cluster methods that fulfil the estimate $\left\|\Psi^{*}\right\|<\varepsilon$ of Assumption II uniformly.

Remark 3.6. Note that the Proof of Theorem 3.4 exhibits the strong link between the $\mathbb{H}^{1}$-ellipticity of the operator $H-E^{*}$ on the orthogonal complement of the eigenspace spanned by $\Psi_{0}$ - and thus the classical quasioptimality estimates for Galerkin discretizations of operator eigenvalue equations, viz., the CI estimate (1.4) on the one hand, and the local strong monotonicity property for the CC function that will deliver similar results for the CC method on the other hand: The lower estimate $H-E^{*} \geq \gamma^{*}$ for the linear operator also constitutes the dominating first order part in the lower estimate (3.6) for $f$, that is then perturbed by the size of the correction $\Psi^{*}$, respectively, the size of the amplitude vector $t^{*}$, so that local strong monotonicity can only fail in the presence of small gaps and/or static correlation.

(iv) Strong monotonicity of $f$ (second characterization). Our second proof for strong monotonicity (Thm. 3.7) circumvents the asumption on the simple eigenvalue of $H$ by assuming that the similarity transformed Hamiltonian can be written as a small perturbation of a one-particle operator, e.g., the Fock operator of the system from unrestricted Hartree-Fock theory. We make two assumptions, introduced in the following.

Assumption BI. Let $\Psi_{0}$ be chosen such that it is an eigenfunction of a one-particle operator constructed from an operator $\bar{F}: H^{1} \rightarrow H^{-1}$

$$
F=\bar{F} \otimes I \otimes \ldots \otimes I+\ldots+I \otimes \ldots \otimes I \otimes \bar{F}
$$

such that

$$
F \Psi_{0}=\Lambda_{0} \Psi_{0},
$$

where $\Lambda_{0}$ is the lowest eigenvalue of $F$ of multiplicity 1 , i.e., there holds

$$
\langle F \Psi, \Psi\rangle \geq \Lambda_{1}\|\Psi\| \quad \text { with } \quad \Lambda_{1}>\Lambda_{0}
$$

for all $\Psi \in \operatorname{span}\left\{\Psi_{0}\right\}^{\perp}$. 
For instance, $\Psi_{0}$ may be chosen as Hartree-Fock or Kohn-Sham ground state. Existence of such ground states has been proven for some Hartree-Fock type functionals in the case that $\sum_{k=1}^{M} Z_{k} \leq N$ in the classical references [58,59], and also recently under the same condition for Kohn-Sham type functionals [1]. For extremely negatively charged molecules, it can be shown that there cannot exist a HF ground state [61].

The nice effect of replacing Assumption AI by Assumption BI is that in the practically relevant case of $\Psi_{0}$ being the (unrestricted) Hartree-Fock determinant, the existence of a HOMO-LUMO gap is guaranteed by the well-known work [7], so that with this canonical choice, Assumption BI and in particular (3.8) is always satisfied. We note also that if spin is regarded, positive definiteness of $F-\Lambda_{0}$ is implied by a spinwise HOMO-LUMO condition [78], about which to our best knowledge not much is known tough.

The second assumption reflecting the regime of perturbation theory is now the following:

Assumption BII. The similarity-transformed Hamiltonian $\mathrm{e}^{-T(t)} H \mathrm{e}^{T(t)}$ may be decomposed into two parts, $\mathrm{e}^{-T(t)} H \mathrm{e}^{T(t)}=\hat{F}(t)+\hat{U}(t)$, where $\hat{F}:=\left(F-\Lambda_{0}\right) T(t)$, with $F-\Lambda_{0}$ the lifted, shifted Fock operator from Assumption BI. Further, we assume the function mapping $\mathbb{V} \rightarrow \mathbb{H}^{-1}, t \mapsto \hat{U}(t) \Psi_{0}$ to be a nonlinear function with small local Lipschitz constant,

$$
\left\|(\hat{U}(t)-\hat{U}(s)) \Psi_{0}\right\|_{\mathbb{H}^{-1}} \leq L \cdot\|t-s\|_{\mathbb{V}}
$$

on a neighborhood $B_{\delta}\left(t^{*}\right)$ of the sought soution $t^{*}$, with $L$ bounded by the HOMO-LUMO gap defined via the constants from Assumption BI,

$$
L<\Lambda_{1}-\Lambda_{0}
$$

The asumption corresponds to te assumption that the Hamiltonian can be well approximated by a Fock-like operator, stressing the perturbational character of Coupled Cluster theory as outlined in the introduction. As the assumption may at first glance appear a bit unnatural, let us comment on it in more detail.

In the appendix, Theorem A.1, it will be shown that the similarity transformed Hamiltonian can be written as an evaluation of at most fourfold commutators, viz., using the decomposition $H=F+U$, we have

$$
\mathrm{e}^{-T} H \mathrm{e}^{T} \Psi_{0}=\left([F, T]+[F, T]_{(2)}+\sum_{n=0}^{4} \frac{1}{n !}[U, T]_{(n)}\right) \Psi_{0},
$$

where $[\cdot, \cdot]_{(n)}$ denotes the $n$-fold commutator. Noting that $[F, T] \Psi_{0}=\left(F-\Lambda_{0}\right) T \Psi_{0}$, this shows that Assumption II indeed means that the terms inferred in the Coupled Cluster function by the fluctuation potential $V$ (and also by the second order terms due to $F$ ) are small compared to the HOMO-LUMO gap. Note that this assumption is related to the basic assumptions under which Rayleigh-Schrödinger perturbation theory [60] can be shown to converge, and also implies the convervence of the fixed point iteration usually used to numerically solve the CC equations (also $c f$. the notes at the end of the Appendix).

This decomposition found, it is now easy to deduce from these assumptions the local strong monotonicity of $f$.

Theorem 3.7 (Local strong monotonicity of the CC function (2)).

Let $f$ denote a $C C$ function based on a reference $\Psi_{0}$ fulfilling Assumption BI and a Hamiltonian $H$ fulfilling Assumption BII. The Coupled Cluster function $f$ then is strongly monotone in a neighbourhood of its solution $t^{*}=\left(t_{\mu}^{*}\right)_{\mu \in \mathcal{M}}$. The constants are uniform for all Coupled Cluster methods for that $\Lambda_{1}-\Lambda_{0}-L$ from BI, BII is uniformly bounded from below.

Proof. The assertion follows with (3.10) from

$$
\begin{aligned}
& \langle f(s)-f(t), s-t\rangle \\
= & \left\langle T(s)-T(t),\left(F-\Lambda_{0}\right)(T(s)-T(t))\right\rangle+\left\langle T(s)-T(t),(\hat{U}(s)-\hat{U}(t)) \Psi_{0}\right\rangle \\
\geq & \left(\left(\Lambda_{1}-\Lambda_{0}\right)-L\right)\|t-s\|_{\mathbb{V}}^{2}=: \quad \gamma\|t-s\|_{\mathbb{V} .}^{2}
\end{aligned}
$$




\section{Existence AND Uniqueness StATEMEnts AND ERROR EStimates}

We now use the just proven properties of $f$ to obtain results about existence and (local) uniqueness for solutions of the problem (2.28) and for discretisations thereof. To this end, we first recall at first that in practice, one at first fixes a discrete one-particle basis $B_{D}$ for the formulation of a specific CC method (e.g. occupied orbitals from a discrete Hartree-Fock or DFT calculation, virtual orbitals orthogonal to the occupied ones), and thus the reference $\Psi_{0}$. From the corresponding Slater basis $\mathbb{B}_{D}$, a subset $\mathbb{B}_{d}$ is selected (e.g. singly and doubly excited Slater determinants for CCSD), corresponding to discrete coefficient spaces $\mathbb{V}_{d} \subseteq \mathbb{V}_{D}$. The equations solved are the projected CC equations

$$
\left\langle f\left(t_{d}\right), v_{d}\right\rangle=0 \text { for all } v_{d} \in \mathbb{V}_{d}
$$

that may, as in (2.29), be interpreted as the continuous $\mathrm{CC}$ equations (2.28) fixed by $\Psi_{0}$ and a basis $\mathbb{B}$ of $\mathbb{H}^{1}$ with $\mathbb{B}_{d} \subseteq \mathbb{B}$, Galerkin-projected on the subspace spanned by $\mathbb{B}_{d}$. This connection will now be used to give statements on the solutions $t^{*}, t_{d}$ of the continuous and discretized equations in Part (i); also, we discuss the results. Part (ii) gives the proof of these results. In (iii), we derive estimates for the Coupled Cluster energy.

(i) Existence and uniqueness of solutions; quasi-optimality of CC. Note that our situation is a little different from what is usually assumed in the theory of standard nonlinear functional analysis [37,89], where existence and uniqueness of continuous as well as discrete solutions follows if $f$ is globally Lipschitz continuous and globally strongly monotone (i.e. (3.1) holds on all of $\mathbb{V}$ ), see, e.g., [33]. This cannot be true in our case if the eigenvalue problem (1.1) has a second solution, corresponding to a bound state aside from the ground state. Instead, existence of the solution of the continuous problem is in our case guaranteed by the gap assumption (2.7) together with Theorem 2.2, and we will prove the existence of local solutions of the corresponding discretised equations using some well-known results from operator analysis. Concerning uniqueness of continuous and discrete solutions, local statements are the best we can hope for if there are bound states aside from the ground state, and a result of that kind is given in the following theorem.

Theorem 4.1 (Existence and uniqueness of solutions; quasi-optimality).

Let the Coupled Cluster function $f$ be strongly monotone at $t^{*}$ (as, e.g., if Assumptions AI and AII, resp., Assumptions BI and BII are fulfilled, see last section). The function $f$ then possesses a Lipschitz continuous inverse $f^{-1}$ on $B_{\delta}\left(t^{*}\right)$ definied in Theorem 3.4; in particular, the solution $t^{*}$ of the Coupled Cluster function that belongs to the lowest eigenvalue of (1.1) is unique in the neighbourhood $B_{\delta}\left(t^{*}\right)$.

Let $\mathbb{V}_{d}$ be a subspace of $\mathbb{V}$ (corresponding to a subspace $\mathbb{H}_{d}^{1}$ of $\mathbb{H}^{1}$ ) for which

$$
d\left(t^{*}, \mathbb{V}_{d}\right):=\min _{v \in \mathbb{V}_{d}}\left\|v-t^{*}\right\|_{\mathbb{V}}=\min _{\Psi \in \mathbb{H}_{d}^{1}}\left\|\Psi-\Psi^{*}\right\|
$$

is sufficiently small. Then the discretised (projected) problem

$$
\left\langle f\left(t_{d}\right), v_{d}\right\rangle=0 \quad \text { for all } \quad v_{d} \in \mathbb{V}_{d}
$$

admits a solution $t_{d}$ in $B_{\delta, d}:=\mathbb{V}_{d} \cap B_{\delta}\left(t^{*}\right)$ which is unique on $B_{\delta, d}$ and fulfils the quasi-optimality estimate

$$
\left\|t_{d}-t^{*}\right\|_{\mathbb{V}} \leq \frac{L}{\gamma} d\left(t^{*}, \mathbb{V}_{d}\right)
$$

with $L$ the Lipschitz constant and $\gamma$ the monotonicity constant of $f$ on $B_{\delta}\left(t^{*}\right)$. In particular, if $\mathbb{V}_{(n)}$ is a sequence of subspaces of $\mathbb{V}$ for which $\lim _{n \rightarrow \infty} d\left(t^{*}, \mathbb{V}_{(n)}\right) \rightarrow 0$, the corresponding solutions $t_{(n)} \in B_{\delta}\left(t^{*}\right) \cap \mathbb{V}_{(n)}$ of (4.2) converge to the continuous solution $t^{*} \in \mathbb{V}$. 


\section{Remarks.}

(i) Theorem 4.1 shows that if the monotonicity constant $\gamma$ is close to zero, which may happen due to a small gap between the ground state energy and the second lowest energy level of the Hamiltonian, or if one is faced with an unsufficient reference determinant, this may not only lead to deterioration of convergence of an, e.g., Newton's method employed for solution of the Coupled Cluster equations as experienced in practice (see, e.g., [43], 13.4.4 for an example with a small energy gap), but also means that the constants in the quasi-optimality estimate (4.3) become bad. Also, the results proven may in this case only hold on a very small neighbourhood of $t^{*}$, emphasing from another viewpoint the importance of multireference approaches in this situation.

(ii) The estimate (4.3) also shows that once a (possibly discrete) reference determinant is fixed, the quality of discrete solutions $t_{d}$ of the discrete CC method is exclusively determined by the choice of the subspace $\mathbb{H}_{d}^{1}$, that is, by the basis functions spanning the virtual space (the space orthogonal to span $B_{\text {occ }}$ ).

A question of particular interest is how the convergence of solutions of discrete CC equations towards the full solution of the electronic Schrödinger equation depends on the solution of the respective discrete Hartree-Fock/DFT equations on which the CC equations are based. The above result implies the following.

Corollary 4.2 (Convergence of combined Hartree-Fock-CC calculations).

Let $\left(\Psi_{0, n}\right)$ a sequence of reference determinants, build from a basis of invariant subspace of respective operators $F_{n}$ according to Assumption $I I$, and $\left(\mathbb{B}_{n}\right)$ a sequence of according bases containing $\Psi_{0, n}$ respectively for all $n \in \mathbb{N}$. If Assumptions AI and AII, respectively, BI and BII, hold uniformly for all $n \in \mathbb{N}$, the corresponding sequence of $C C$ equations,

$$
f_{n}(t)=\left\langle\Psi_{\mu}, \mathrm{e}^{-T(t)} H \mathrm{e}^{T(t)} \Psi_{0, n}\right\rangle=0 \quad \text { for all } \Psi_{\mu} \in \mathbb{B}_{n}
$$

for $f_{n}: \mathbb{V}_{n} \rightarrow \mathbb{V}_{n}^{\prime}$ admits a sequence of solutions $\left(t_{n}\right)_{n \in \mathbb{N}}, t_{n} \in \mathbb{V}_{n} \cap B_{\delta}\left(t^{*}\right)$, which for each $n$ are unique on $\mathbb{V}_{n} \cap B_{\delta}\left(t^{*}\right)$ respectively, and which uniformly fulfil the estimate

$$
\left\|t_{d}-t^{*}\right\|_{\mathbb{V}} \leq \frac{L}{\gamma} d\left(t^{*}, \mathbb{V}_{n}\right) \lesssim \bar{d}
$$

Proof. The corollary follows from Theorem 4.1 together with the fact that once the constants $\bar{\varepsilon}, \bar{d}$ can be chosen uniformly, all estimates in this work hold uniformly (provided the Hamiltonian $H$ is fixed).

The above Corollary 4.2 assumes that the reference determinants $\Psi_{0, n}$ are close enough to the true solution $\underline{\Psi}$. Asymptotically, this is in particular the case if the sequence of reference determinants converges to a continuous limit $\Psi_{0 \text {,cont }}$ fulfilling this condition. Unfortunately, for the canonical choice of building $\Psi_{0}$ from the solution of the discrete Hartree-Fock equations, the convergence towards the continuous solution is an open question. See [18] for a recent analysis of a related model problem.

(ii) Proof of Theorem 4.1. Equation (3.1) implies that $f$ is one-to-one on $B_{\delta}\left(t^{*}\right)$ and that for $p, q \in f\left(B_{\delta}\left(t^{*}\right)\right)$, there holds for the inverse mapping $f^{-1}: f\left(B_{\delta}\left(t^{*}\right)\right) \rightarrow B_{\delta}\left(t^{*}\right)$ that

$$
\gamma\left\|f^{-1} p-f^{-1} q\right\|_{\mathbb{V}}^{2} \leq\left\langle p-q, f^{-1} p-f^{-1} q\right\rangle_{\ell_{2}} \leq\|p-q\|_{\mathbb{V}^{\prime}}\left\|f^{-1} p-f^{-1} q\right\|_{\mathbb{V}},
$$

so $f^{-1}$ is Lipschitz continuous with Lipschitz constant $1 / \gamma$. To prove the existence of solutions for sufficiently well discretised problems, we use the following well-known lemma which bases on the fixed point theorem of Brouwder, see, e.g., [33], Lemma 4.2.1 for a proof.

Lemma 4.3. Let $\|\cdot\|_{\#}$ be an arbitrary norm on $\mathbb{R}^{m}$, and $\mathbf{h}: \mathbb{R}^{m} \rightarrow \mathbb{R}^{m}$ be a continuous function on the closed ball $\bar{B}_{R,\|\cdot\|_{\#}}(\mathbf{0})$ of radius $R$ around $\mathbf{0} \in \mathbb{R}^{m}$. If $\langle\mathbf{h}(\mathbf{v}), \mathbf{v}\rangle \geq 0$ holds for all $\mathbf{v} \in \mathbb{R}^{m}$ with $\|\mathbf{v}\|_{\#}=R$, there is a $\mathbf{v}^{*} \in \bar{B}_{R,\|.\| \#}(\mathbf{0})$ for which $\mathbf{h}\left(\mathbf{v}^{*}\right)=\mathbf{0}$. 
To finish the Proof of Theorem 4.1, let us now fix a discretisation $\mathbb{V}_{d} \subseteq \mathbb{V}$ for which $\kappa:=d\left(t^{*}, \mathbb{V}_{d}\right) \leq \delta \cdot \gamma /(\gamma+L)$. We let $m:=\operatorname{dim} \mathbb{V}_{d}, \mathbb{B}_{d}:=\left\{b_{j} \in \mathbb{V}, 1 \leq j \leq m\right\}$ be an orthonormal basis of $\mathbb{V}_{d}$ and $t^{\text {opt }}=\arg \min d\left(t^{*}, \mathbb{V}_{d}\right) .{ }^{5}$ To apply Lemma 4.3 , we define for $\mathbf{v}=\left(v_{j}\right)_{j=1}^{m} \in \mathbb{R}^{m}$ that $v=\sum_{j=1}^{m} v_{j} b_{j}$ and $\|\mathbf{v}\|_{\#}:=\|v\|_{\mathbb{V}}$. We let

$$
\mathbf{h}(\mathbf{v})=\left(\left\langle f\left(t^{\mathrm{opt}}+\sum_{j=1}^{m} v_{j} b_{j}\right), b_{k}\right\rangle\right)_{k=1}^{m}
$$

and observe that $\mathbf{h}(\mathbf{v})=\mathbf{0}$ for some $\mathbf{v} \in \bar{B}_{R, \#}(\mathbf{0})$ iff $t^{\mathrm{opt}}+\sum_{j=1}^{m} v_{j} b_{j} \in \bar{B}_{R, \mathbb{V}}\left(t^{\mathrm{opt}}\right)$ solves the discretised problem (4.2). Choosing $R=\delta-\kappa, \bar{B}_{R, \mathbb{V}}\left(t^{\mathrm{opt}}\right)$ lies in the neighbourhood of $t^{*}$ where $f$ is strongly monotone, so for all $\mathbf{v} \in \mathbb{R}^{m}$ with $\|\mathbf{v}\|_{\#}=R$,

$$
\begin{aligned}
\langle\mathbf{h}(\mathbf{v}), \mathbf{v}\rangle & :=\left\langle f\left(t^{\mathrm{opt}}+v\right), v\right\rangle \\
& =\left\langle f\left(t^{\mathrm{opt}}+v\right)-f\left(t^{\mathrm{opt}}\right), v\right\rangle+\left\langle f\left(t^{\mathrm{opt}}\right)-f\left(t^{*}\right), v\right\rangle \\
& \geq \gamma\|\mathbf{v}\|_{\#}^{2}-L \kappa\|\mathbf{v}\|_{\#}=R(\gamma R-L \kappa) \geq 0
\end{aligned}
$$

and because of the local Lipschitz continuity of $f, \mathbf{h}$ is continuous on $\bar{B}_{R}(0)$. Thus, $\mathbf{h}$ fulfils the conditions of Lemma 4.3 , and if $\mathbf{v}^{*}=\left(v_{j}^{*}\right)_{j=1}^{m} \in \bar{B}_{R, \#}(\mathbf{0})$ solves $\mathbf{h}\left(\mathbf{v}^{*}\right)=\mathbf{0}$, then $t_{d}:=t^{\mathrm{opt}}+\sum_{j=1}^{m} v_{j}^{*} b_{j} \in \bar{B}_{R, \mathbb{v}}\left(t^{\mathrm{opt}}\right) \subseteq B_{\delta}\left(t^{*}\right)$ is a solution of (4.2). The restriction $\tilde{f}: B_{d, \delta}\left(t^{*}\right) \rightarrow \mathbb{V}_{d}^{\prime}$ of $f$ is also a strongly monotone function, so that with the same argumentation as for the continuous solution, there can only be one $t_{d} \in B_{d, \delta}\left(t^{*}\right)$ solving $\tilde{f}\left(t_{d}\right)=0 \in \mathbb{V}_{d}^{\prime}$, proving local uniqueness of the solution. The quasi-optimality estimate and the convergence of the discrete solutions $t_{d}$ towards the continuous limit $t^{*}$ now follow from Lipschitz continuity and strong monotonicity of $f$ by standard arguments, see, e.g., [89].

(iii) Error estimates for the Coupled Cluster energy. To end this section, we now combine the results just proven with the formalism of goal oriented error estimators developed in [13] and also used in [82] to obtain estimators for the Coupled Cluster energy (2.26),

$$
E(t)=\left\langle\Psi_{0}, \mathrm{e}^{-T(t)} H \mathrm{e}^{T(t)} \Psi_{0}\right\rangle,
$$

in terms of the approximation quality of the cluster amplitudes $\left(t_{\mu}\right)_{\mu \in \mathcal{M}}$ and the corresponding wave functions. To do so, we use that the ground state energy $E^{*}$ is a minimizer of a corresponding Lagrange functional. Because this Lagrangian is non-symmetric, we cannot expect the error to be quadratic with respect to the error of the wave function as for the energies obtained, e.g., by Hartree-Fock or CI calculations. Instead, the solution $z^{*}$ of the dual problem (corresponding to the Lagrangian multipliers in the finite-dimensional setting) enters the estimates, and we introduce the necessary terminology in the next lemma.

Lemma 4.4 (Properties of dual solutions). Let $\mathbb{V}_{d}$ be a sufficiently good subspace of $\mathbb{V}$, and $t^{*} \in \mathbb{V}$ and $t_{d} \in \mathbb{V}_{d}$ solutions the Coupled Cluster equations (2.26) and of the discretised (projected) Coupled Cluster equations respectively,

$$
\left\langle f\left(t^{*}\right), s\right\rangle=0 \quad \text { for all } \quad s \in \mathbb{V}, \quad\left\langle f\left(t_{d}\right), s_{d}\right\rangle=0 \quad \text { for all } \quad s_{d} \in \mathbb{V}_{d}
$$

If $f$ is strongly monotone at $t^{*}$, there is a unique "dual solution" or "Lagrangian multiplier" $z^{*} \in \mathbb{V}$ determined by $t^{*}$ such that $\left(t^{*}, z^{*}\right)$ is a stationary point of the Lagrangian $\mathcal{L}(t, z)=E(t)+\langle f(t), z\rangle$, i.e. $\left(t^{*}, z^{*}\right)$ solves

$$
\mathcal{L}^{\prime}\left(t^{*}, z^{*}\right)=\left\{\begin{array}{c}
\left\langle E^{\prime}\left(t^{*}\right), s\right\rangle-\left\langle D f\left(t^{*}\right) s, z^{*}\right\rangle \\
\left\langle f\left(t^{*}\right), s\right\rangle
\end{array}\right\}=0 \quad \text { for all } s \in \mathbb{V} .
$$

\footnotetext{
${ }^{5}$ Note that $\mathbb{V}$ is a Hilbert space, see the remarks after $(2.20)$.
} 
For a sufficiently good discretisation $\mathbb{V}_{d}$, there is a corresponding unique $z_{d} \in \mathbb{V}$ such that $\left(t_{d}, z_{d}\right)$ solves the discretised equations

$$
\mathcal{L}^{\prime}\left(t_{d}, z_{d}\right)=\left\{\begin{array}{c}
\left\langle E^{\prime}\left(t_{d}\right), s_{d}\right\rangle-\left\langle D f\left(t_{d}\right) s_{d}, z_{d}\right\rangle \\
\left\langle f\left(t_{d}\right), s_{d}\right\rangle
\end{array}\right\}=0 \quad \text { for all } s_{d} \in \mathbb{V}_{d}
$$

The discrete dual solution $z_{d}$ approximates the exact dual solution quasi-optimally in the sense that

$$
\left\|z_{d}-z^{*}\right\|_{\mathbb{V}} \lesssim \Delta:=\max \left\{d\left(\mathbb{V}_{d}, t^{*}\right), d\left(\mathbb{V}_{d}, z^{*}\right)\right\}
$$

Proof. By definition, $t^{*}$ solves the second equation from (4.7), so we only have to show that the first equation $\left\langle E^{\prime}\left(t^{*}\right), s\right\rangle=\left\langle D f\left(t^{*}\right) s, z^{*}\right\rangle$ admits a unique solution $z^{*}$. Indeed, this is an equation for the linear operator $D f\left(t^{*}\right)^{\dagger}: \mathbb{V} \rightarrow \mathbb{V}^{\prime}$, which is bounded and coercive because its adjoint $D f\left(t^{*}\right)$ is by Corollary 3.3. Therefore, the Lax-Milgram theorem (see, e.g., [3]) ensures existence and uniqueness of $z^{*}$. The same argument holds for $z_{d}$ if the discretisation is fine enough to guarantee (together with quasi-optimality of $t_{d}$ ) that $\operatorname{Df}\left(t_{d}\right)$ is also coercive, cf. Corollary 3.3. To show (4.9), we decompose $z_{d}-z^{*}=z_{d}-\hat{z}_{d}+\hat{z}_{d}-z^{*}$, where $\hat{z}_{d} \in \mathbb{V}_{d}$ solves the discrete system

$$
\left\langle E^{\prime}\left(t^{*}\right), s_{d}\right\rangle=\left\langle D f\left(t^{*}\right) s_{d}, \hat{z}_{d}\right\rangle \quad \text { for all } \quad s_{d} \in \mathbb{V}_{d}
$$

Because $D f\left(t^{*}\right)$ is a bounded and coercive linear mapping, see Corollary 3.3, $\hat{z}_{d}$ approximates the solution $z^{*}$ of the corresponding continuous problem (4.7) quasi-optimally by Cea's Lemma [3], $\left\|\hat{z}_{d}-z^{*}\right\|_{\mathbb{V}} \lesssim d\left(\mathbb{V}_{d}, z^{*}\right)$. For $\left\|z_{d}-\hat{z}\right\|_{\mathbb{V}}$, we at first note again that $D f(t)$ and also by very similar arguments the derivative $E^{\prime}(t)$ of the energy expression (4.5) are Lipschitz continuous on bounded neighbourhoods of $t^{*}$. We choose $c>0$ such that by Theorem 4.1, for a each discretisation $\mathbb{V}_{d}$ for which $d\left(t^{*}, \mathbb{V}_{d}\right)<c$ there holds $\left\|t_{d}-t^{*}\right\|_{\mathbb{V}} \leq L / \gamma d\left(t^{*}, \mathbb{V}_{d}\right)$ for the discrete solution $t_{d}$, and let $L_{f^{\prime}}$ and $L_{E^{\prime}}$ be the Lipschitz constants of $D f(t)$ and $E^{\prime}(t)$ on $B_{c L / \gamma}\left(t^{*}\right)$. We now obtain using (4.8), (4.10) that

$$
\begin{aligned}
\gamma\left\|z_{d}-\hat{z}_{d}\right\|_{\mathbb{V}}^{2} & \leq\left\langle D f\left(t_{d}\right)\left(z_{d}-\hat{z}_{d}\right), z_{d}-\hat{z}_{d}\right\rangle \\
& =\left\langle E^{\prime}\left(t_{d}\right)-E^{\prime}\left(t^{*}\right),\left(z_{d}-\hat{z}_{d}\right)\right\rangle+\left\langle\left(D f\left(t^{*}\right)-D f\left(t_{d}\right)\right)\left(z_{d}-\hat{z}_{d}\right), \hat{z}_{d}\right\rangle \\
& \leq\left(L_{E^{\prime}}+L_{f^{\prime}}\|\hat{z}\|_{\mathbb{V}}\right)\left\|t_{d}-t^{*}\right\|_{\mathbb{V}}\left\|z_{d}-\hat{z}_{d}\right\|_{\mathbb{V}}
\end{aligned}
$$

and observe that $\|\hat{z}\|_{\mathbb{V}}$ is bounded by $\left\|z^{*}\right\|_{\mathbb{V}}+c \cdot d\left(\mathbb{V}_{d}, z^{*}\right)$, so that

$$
\left\|z_{d}-\hat{z}_{d}\right\|_{\mathbb{V}} \lesssim\left\|t_{d}-t^{*}\right\|_{\mathbb{V}} \lesssim d\left(\mathbb{V}_{d}, t^{*}\right)
$$

Thus, $\left\|z_{d}-z^{*}\right\|_{\mathbb{V}} \lesssim \Delta$, finishing the proof.

The quality of a discrete solution $\left(t_{d}, z_{d}\right)$ of the above Lagrangian equations can be measured in terms of the primal residual $\rho\left(t_{d}\right)$ and the dual residual $\rho^{*}\left(t_{d}, z_{d}\right)$, given by

$$
\rho\left(t_{d}\right):=\left\langle f\left(t_{d}\right), \cdot\right\rangle_{\mathbb{V}} \quad \rho^{*}\left(t_{d}, z_{d}\right):=\left\langle E^{\prime}\left(t_{d}\right), \cdot\right\rangle_{\mathbb{V}}-\left\langle D f\left(t_{d}\right) \cdot, z_{d}\right\rangle_{\mathbb{V}}
$$

The theory developed in $[9,13]$ now allows to estimate the error of the energy approximation in terms of these primal and dual residuals. We first adapt the original theorem from [9] to our notation in (i) and then derive some quasi-optimality estimates for the Coupled Cluster method in (ii), (iii). The estimates again hold uniformly for different $\mathrm{CC}$ functions based on different reference determinants $\Psi_{0}$ and bases $\mathbb{B}$ if the conditions given in Corollary 4.2 are satisfied. 
Theorem 4.5 (Energy estimators).

(i) (Becker/Rannacher [13], see [9] Prop. 6.2).

Let $\left(t^{*}, z^{*}\right) \in \mathbb{V}^{2}$ and $\left(t_{d}, z_{d}\right) \in \mathbb{V}_{d}^{2}$ be the solutions of minimization problems (4.7), (4.8) for a thrice differentiable functional $\mathcal{L}$. Then there holds

$$
E\left(t^{*}\right)-E\left(t_{d}\right)=\frac{1}{2} \rho\left(t_{d}\right)\left(z^{*}-v_{d}\right)+\frac{1}{2} \rho^{*}\left(t_{d}, z_{d}\right)\left(t^{*}-w_{d}\right)+\mathcal{R}_{d}^{3}
$$

for all $v_{d}, w_{d}$ in $\mathbb{V}_{d}$, where

$$
\mathcal{R}_{d}^{3}=\mathcal{O}\left(\max \left\{\left\|t^{*}-t_{d}\right\|_{\mathbb{V}},\left\|z^{*}-z_{d}\right\|_{\mathbb{V}}\right\}^{3}\right)
$$

depends cubically on the primal and dual errors.

(ii) Let $\mathbb{V}_{d}$ be a sufficiently large subspace of $\mathbb{V}$ in the sense that for $\Delta$ from (4.9), $\Delta<c$ for a suitable $c>0$, and denote by $\left(t^{*}, z^{*}\right)$ and $\left(t_{d}, z_{d}\right)$ the solutions the Coupled Cluster equations and of the discretised (projected) Coupled Cluster equations (4.6), respectively, together with the corresponding unique dual solutions. If the Coupled Cluster function $f$ is strongly monotone at $t^{*}$, there holds

$$
\begin{gathered}
\left|E\left(t^{*}\right)-E\left(t_{d}\right)\right| \leq\left\|t_{d}-t^{*}\right\|_{\mathbb{V}}\left(c_{1}\left\|t_{d}-t^{*}\right\|_{\mathbb{V}}+c_{2}\left\|z_{d}-z^{*}\right\|_{\mathbb{V}}\right), \\
\left|E\left(t^{*}\right)-E\left(t_{d}\right)\right| \lesssim\left(d\left(\mathbb{V}_{d}, t^{*}\right)+d\left(\mathbb{V}_{d}, z^{*}\right)\right)^{2} .
\end{gathered}
$$

where the above constants are specified in the proof.

(iii) Denoting $\underline{\Psi}^{z^{*}}:=\Psi_{0}+\Psi^{z^{*}}:=\mathrm{e}^{T\left(z^{*}\right)} \Psi_{0}$, by $\underline{\Psi}=\Psi_{0}+\Psi^{*}$ the solution of the exact eigenproblem (1.1) and by $\mathbb{H}_{d, \perp}^{1}$ the discretisation of $\left(\operatorname{span}\left\{\Psi_{0}\right\}\right)^{\perp}$ corresponding to $\mathbb{V}_{d}$, there holds

$$
\begin{gathered}
\left|E\left(t^{*}\right)-E\left(t_{d}\right)\right| \lesssim\left\|\mathrm{e}^{T\left(t_{d}\right)} \Psi_{0}-\underline{\Psi}\right\| \cdot\left(\left\|\mathrm{e}^{T\left(t_{d}\right)} \Psi_{0}-\underline{\Psi}\right\|^{2}+\left\|\mathrm{e}^{T\left(z_{d}\right)} \Psi_{0}-\underline{\Psi}^{z^{*}}\right\|\right), \\
\left|E\left(t^{*}\right)-E\left(t_{d}\right)\right| \lesssim\left(\inf _{\Psi \in \mathbb{H}_{d, \perp}^{1}}\left\|\Psi-\Psi^{*}\right\|+\inf _{\Psi \in \mathbb{H}_{d, \perp}^{1}}\left\|\Psi-\Psi^{z^{*}}\right\|\right)^{2} .
\end{gathered}
$$

Proof. For the proof of (i), cf. [9]. To prove (ii), we choose $\tilde{c}>0$ such that for a each discretisation $\mathbb{V}_{d}$ for which $d\left(t^{*}, \mathbb{V}_{d}\right)<\tilde{c}$, there holds $\left\|t_{d}-t^{*}\right\|_{\mathbb{V}} \leq L / \gamma d\left(t^{*}, \mathbb{V}_{d}\right)$ for the discrete solution $t_{d}$ by Theorem 4.1. We denote by $L, L_{f^{\prime}}$ and $L_{E^{\prime}}$ the Lipschitz constants of $f(t), D f(t)$ and $E^{\prime}(t)$ on $B_{\tilde{c} L / \gamma}\left(t^{*}\right)$, and note that by Corollary 3.3 , $\|D f(t)\|_{\mathbb{V} \rightarrow \mathbb{V}^{\prime}}$ is uniformly bounded by a constant $C$ on $B_{\tilde{c} L / \gamma}\left(t^{*}\right)$. We now use (4.7) to rewrite the dual residual by inserting zeros as

$$
\rho^{*}\left(t_{d}, z_{d}\right)(s)=\left\langle E^{\prime}\left(t_{d}\right)-E^{\prime}\left(t^{*}\right), s\right\rangle_{\ell_{2}}+\left\langle\left(D f\left(t^{*}\right)-D f\left(t_{d}\right)\right) s, z^{*}\right\rangle_{\ell_{2}}+\left\langle D f\left(t_{d}\right) s, z^{*}-z_{d}\right\rangle_{\ell_{2}}
$$

for arbitrary $s \in \mathbb{V}$. Thus, with (4.12) and the definition of the primal residual $\rho\left(t_{d}\right)$, we obtain that for all $v_{d}$, $w_{d}$ in $\mathbb{V}_{d}$, there holds according to (i) that

$$
\begin{aligned}
& 2\left|E\left(t^{*}\right)-E\left(t_{d}\right)\right| \\
\leq & \left|\left\langle f\left(t_{d}\right)-f\left(t^{*}\right), z^{*}-v_{d}\right\rangle_{\ell_{2}}\right|+\left|\left\langle E^{\prime}\left(t_{d}\right)-E^{\prime}\left(t^{*}\right), t^{*}-w_{d}\right\rangle_{\ell_{2}}\right| \\
& +\left|\left\langle\left(D f\left(t^{*}\right)-D f\left(t_{d}\right)\right)\left(t^{*}-w_{d}\right), z^{*}\right\rangle_{\ell_{2}}\right|+\left|\left\langle D f\left(t_{d}\right)\left(t^{*}-w_{d}\right), z^{*}-z_{d}\right\rangle_{\ell_{2}}\right|+2 \mathcal{R}_{d}^{3} \\
\leq & L\left\|t_{d}-t^{*}\right\| \mathbb{V}\left\|z^{*}-v_{d}\right\|_{\mathbb{V}}+L_{E^{\prime}}\left\|t_{d}-t^{*}\right\|_{\mathbb{V}}\left\|t^{*}-w_{d}\right\|_{\mathbb{V}} \\
& +L_{f^{\prime}}\left\|t^{*}-t_{d}\right\|_{\mathbb{V}}\left\|t^{*}-w_{d}\right\|_{\mathbb{V}}\left\|z^{*}\right\|_{\mathbb{V}}+C\left\|t^{*}-w_{d}\right\|_{\mathbb{V}}\left\|z^{*}-z_{d}\right\|_{\mathbb{V}}+2 \mathcal{R}_{d}^{3}:=(*) .
\end{aligned}
$$

Inserting $v_{d}=t_{d}, w_{d}=z_{d}$, we obtain

$$
\begin{aligned}
\left|E\left(t^{*}\right)-E\left(t_{d}\right)\right| & \leq \frac{1}{2}\left\|t_{d}-t^{*}\right\|_{\mathbb{V}}\left(\left(L_{f^{\prime}}\left\|z^{*}\right\|_{\mathbb{V}}+L_{E^{\prime}}\right)\left\|t_{d}-t^{*}\right\|_{\mathbb{V}}+(L+C)\left\|z_{d}-z^{*}\right\|_{\mathbb{V}}\right)+\mathcal{R}_{d}^{3} \\
& =:\left\|t_{d}-t^{*}\right\|_{\mathbb{V}}\left(c_{1}\left\|t_{d}-t^{*}\right\|_{\mathbb{V}}+c_{2}\left\|z_{d}-z^{*}\right\|_{\mathbb{V}}\right)+\mathcal{R}_{d}^{3}
\end{aligned}
$$


By Lemma 4.4, $\left\|z_{d}-z^{*}\right\|_{\mathbb{V}}$ is bounded by $\Delta$; thus, we can (by additionally using the quasi-optimality of $t_{d}$, Thm. 4.1) control the remainder term $\mathcal{R}_{d}^{3}$ in terms of $\mathcal{O}\left(\Delta^{3}\right)$. Therefore, the first estimate of (ii) is proven by choosing $\Delta$ small enough, while the second follows from $(*)$ by inserting for $v_{d}, w_{d}$ the best approximations $t^{\mathrm{opt}}, z^{\mathrm{opt}} \in \mathbb{V}_{d}$ of $t^{*}, z^{*}$. To prove (iii), we use Lemma 2.3: The first estimate follows from the first one of (ii) with the observation that locally,

$$
\|t-s\|_{\mathbb{V}}=\left\|T(t) \Psi_{0}-T(s) \Psi_{0}\right\| \sim\left\|\mathrm{e}^{T(t)} \Psi_{0}-\mathrm{e}^{T(s)} \Psi_{0}\right\|
$$

holds; for the second, we use that $\left\{\exp (T(t)) \mid t \in \mathbb{V}_{d}\right\}=\left\{I+T(t) \mid t \in \mathbb{V}_{d}\right\}$, cf. also Lemma 2.3, together with the second estimate given in (ii).

\section{Concluding Remarks}

In this paper, we used the infinite dimensional, continuous formulation of the Coupled Cluster method derived in [79] to apply to it concepts of nonlinear operator theory along the lines of [82], and this ansatz has enabled us to formulate direct error estimates with respect to the continuous solution $\underline{\Psi}$. In particular, the equations obey similar estimates as the corresponding full-CI-equations, with the Galerkin-typical quadratic dependence of the eigenvalues on the error of the iterates replaced by the nonvariational dual estimate from (4.5). These estimates provide a tool that might be used for error estimation with an appropriate refinement strategy, for instance by using hierarchical basis sets as the VnZ-bases used in extrapolation schemes, or also by selecting subsets of a discretised set of amplitudes to estimate the effect of including, e.g., only some of the $T_{2}$ amplitudes in a classical CCSD calculation.

Our analysis also displays the importance of the spectral gap (3.4) and of the quality of the reference determinant not only for the convergence of the method, but also for the constants in optimality estimates. If these quantities are well-behaved, the proof of Theorem 3.4 shows that the CC function can then be viewed as a small perturbation of the symmetric operator $H-E^{*}$. Continuity arguments imply in this case that primal and dual solution do not differ much, so that if $d\left(z^{*}, \mathbb{V}_{d}\right) \lesssim d\left(t^{*}, \mathbb{V}_{d}\right)$ holds, the CC energy converges quadratically with the error in $t^{*}$ - a behaviour that may often be observed in practice for well-behaved examples. On the other hand, our analysis also underlines the general weakness of the practical Coupled Cluster method if the spectral gap (3.4) is too small or if multiple eigenvalues occur, or if the reference determinant $\Psi_{0}$ is of poor quality. In this case, multireference methods or have to be utilized, and it would be desirable to use the theoretical framework developed here to attack this problem and also the approaches of time dependent CC and variational CC (see, e.g., [53] and references therein) from the viewpoint of numerical analysis in the near future.

\section{Appendix A. Practical aspects of the CC method}

In this final supplementary section, we want to give a brief overview on how the continuous CC equations (2.26), respectively, their projected versions (2.30), are treated in practice. As a first step towards implementation, the CC equations are simplified by rewriting the term $\mathrm{e}^{-T(t)} H \mathrm{e}^{T(t)}$ by means of the Baker-Campbell-Hausdorff formula, giving a series expansion which terminated at fourth order due to the structure of the Hamiltonian. Here, we will give a short proof of this fact that to the authors' mind more transparent than the canonical one that can, e.g., be found in [43]. The according expression for the similarity transformed Hamiltonian $\mathrm{e}^{-T} H \mathrm{e}^{T}$ (with $T=T(t)$, also covering the continuous case) will be given in part (i), Theorem A.1, while using the formalism of second quantization, the main part of the proof is performed in (ii) and (iii). Afterwards, we will in (iv) only make some minor remarks about how the resulting terms are evaluated in practice and refer the reader to the literature for further reference. In particular, we will not try to compete with the vast amount of practical experience made with the CC method in the field of quantum chemistry; for numerical examples for the performance of the CC method, we therefore refer to [43] and the references given therein. The section will be ended in part (v) by a uniform convergence estimate for Newton's method applied to the CC equations, which we trivially obtain from the strong monotonicity results for $f$. 
(i) Formulation of CC in terms of annihilation and creation operators. We already mentioned above that the projected Coupled Cluster equations (i.e. the term $\mathrm{e}^{-T} H \mathrm{e}^{T}$ ) can be evaluated exactly within arithmetic precision. This is due to the fact that in the linked Coupled Cluster equations (2.26), the term $\mathrm{e}^{-T} H \mathrm{e}^{T}$ can be expanded into the so-called Baker-Campbell-Hausdorff series, which itself terminates because the Hamiltonian is a two-particle operator [43]. To state the result precisely, we define for any operator $A: H^{1} \rightarrow H^{-1}$ the (iterated) commutators $[A, T]_{(0)}:=A,[A, T]_{(1)}:=A T-T A: H^{1} \rightarrow H^{-1}$ and $[A, T]_{(n)}:=\left[[A, T]_{(n-1)}, T\right]$ for $n \geq 2$, and note that these expressions are well-defined due to Theorem 2.1.

Theorem A.1 (Evaluation of the similarity transformed Hamiltonian $\mathrm{e}^{-T} H \mathrm{e}^{T}$ ). For the Hamiltonian $H: \mathbb{H}^{1} \rightarrow \mathbb{H}^{-1}$, there holds

$$
\mathrm{e}^{-T} H \mathrm{e}^{T}=\sum_{n=0}^{4} \frac{1}{n !}[H, T]_{(n)} .
$$

The first part of the proof is the below globalization of the Baker-Campbell-Hausdorff series expansion (as canonically formulated for matrices). Afterwards, we show in (ii), (iii) that the iterated commutators $[H, T]_{(n)}$ give zero contributions for $n>4$.

Lemma A.2. For any operator $A: H^{1} \rightarrow H^{-1}$, there holds the Baker-Campbell-Hausdorff formula,

$$
\mathrm{e}^{-T} A \mathrm{e}^{T}=\sum_{n=0}^{\infty} \frac{1}{n !}[A, T]_{(n)} .
$$

Proof. It is not hard to show by induction that $[A, T]_{(n)}=\sum_{i=0}^{n}\left(\begin{array}{c}n \\ i\end{array}\right)(-1)^{i} T^{i} A T^{n-i}$. Thus,

$$
\mathrm{e}^{-T} A \mathrm{e}^{T}=\sum_{i=0}^{\infty} \sum_{j=0}^{\infty} \frac{(-T)^{i}}{i !} A \frac{T^{j}}{j !}=\sum_{n=0}^{\infty} \sum_{i=0}^{n}(-1)^{i} \frac{T^{i}}{i !} A \frac{T^{n-i}}{(n-i) !}=\sum_{n=0}^{\infty} \frac{1}{n !}[A, T]_{(n)} .
$$

(ii) The CC equations in Second Quantization. The fact that the Baker-Campbell-Hausdorff series terminates can be best understood in terms of annihilation and creation operators borrowed the formalism of Second Quantization. We therefore quickly repeat some facts from [79], where we globalized the canonical definition via a finite-dimensional basis $[43,85]$ to the full space $\mathbb{L}^{2}$. For a more detailed treatment, see the afore mentioned references. At the end of the present part (ii), we obtain an expression for the similarity transformed Hamiltonian $\mathrm{e}^{-T} H \mathrm{e}^{T}$ in terms of annihilation and creation operators.

We start by defining the (fermionic) Fock space [35] as

$$
\mathbb{F}:=\bigoplus_{i=0}^{\infty} \mathbb{L}_{i}^{2}
$$

where $\bigoplus$ denotes the direct orthogonal sum, and $\mathbb{L}_{i}^{2}:=L^{2}\left(\left(\mathbb{R}^{3} \times\left\{ \pm \frac{1}{2}\right\}\right)^{i}\right)$ denotes the $i$-electron state space. In particular, $\mathbb{L}^{2}=\mathbb{L}_{N}^{2}$ is embedded in $\mathbb{F}$. As before, we fix a one-particle basis $B=\left\{\chi_{P} \mid P \in \mathbb{N}\right\}$. For each $\chi_{P} \in B$, we define a corresponding creation operator $a_{P}^{\dagger}$ on the Fock space $\mathbb{F}$. Its action on an $i$-electron Slater determinant $\Psi_{\mu, i} \in \mathbb{B}_{i}$ can be described loosely as adding to $\Psi_{\mu, i}$ the basis function $\chi_{P}$ to obtain an antisymmetric $(i+1)$-electron Slater determinant with a certain sign \pm 1 ; if $\chi_{P}$ is contained in $\Psi_{\mu, i}$, the action of $\mathcal{Q}_{i+1}$ results in $a_{P}^{\dagger} \Psi_{\mu, i}=0$. Formally and more precisely, this is expressed by the formula

$$
a_{P}^{\dagger} \Psi_{\mu, i}:=\frac{\sqrt{N !}}{\sqrt{(N+1) !}} \sum_{\pi \in S(N)}(-1)^{\operatorname{sgn}(\pi)}\left[\chi_{P}\left(x_{\pi(1)}, s_{\pi(1)}\right) \otimes \Psi_{\mu, i}\left(x_{\pi(2)}, s_{\pi(2)}, \ldots, x_{\pi(N)}, s_{\pi(N)}\right)\right]
$$


with the sum running over the permutational group $S(N)$ on $N$ elements operating on the indices of $\Psi$ analogously to the construction (2.8) for the Slater determinants. For $N=0$, we let $a_{P}^{\dagger}|\rangle=\chi_{P} \in \mathbb{L}_{1}^{2}$. By linear continuation and closing the operator in $\mathbb{F}$, the definition of $a_{P}^{\dagger}$ yields a bounded operator defined on all of $\mathbb{F}$. The corresponding annihilation operator $a_{P}: \mathbb{F} \rightarrow \mathbb{F}$ of $\chi_{P}$ is defined as the adjoint of $a_{P}^{\dagger}: \mathbb{F} \rightarrow \mathbb{F}$; it "removes" $\chi_{P}$ from the Slater determinant $\Psi_{\mu, i}$ to give an $(i-1)$-electron Slater determinant, or returns $a_{P}^{\dagger} \Psi_{\mu, i}=0$ if $\chi_{P}$ is not contained in $\Psi_{\mu, i}$.

Among many other properties $[43,85]$ of annihilation and creation operators, the probably most important are the rules for computation of their anticommutators $[A, B]_{+}:=A B+B A$,

$$
\left[a_{P}, a_{Q}\right]_{+}=0, \quad\left[a_{P}^{\dagger}, a_{Q}^{\dagger}\right]_{+}=0, \quad\left[a_{P}, a_{Q}^{\dagger}\right]_{+}=\left[a_{P}^{\dagger}, a_{Q}\right]_{+}=\delta_{P, Q}
$$

for all $P, Q \in \mathbb{N}$, where $\delta_{P, Q}=1$ only if $P=Q$ and $\delta_{P, Q}=0$ otherwise, and we will now use these properties to simplify the expression $\mathrm{e}^{-T} H \mathrm{e}^{T}$ turning up in the CC equations. We therefore use that the Hamiltonian $H$ can be expressed [78] in Second Quantization form as

$$
H=\sum_{P, Q \in \mathbb{N}} h_{P Q} a_{P}^{\dagger} a_{Q}+\sum_{P, Q, R, S \in \mathbb{N}}\langle P Q \| R S\rangle a_{P}^{\dagger} a_{Q}^{\dagger} a_{S} a_{R}
$$

with the single electron interaction integrals

$$
h_{P Q}:=\frac{1}{2}\left\langle\nabla \chi_{P}, \nabla \chi_{Q}\right\rangle+\sum_{\nu=1}^{K}\left\langle\chi_{P}, \frac{Z_{\nu}}{\left|x_{i}-R_{\nu}\right|} \chi_{Q}\right\rangle
$$

and the electron pair interaction integrals

$$
\langle P Q \mid R S\rangle:=\sum_{s, s^{\prime} \in\left\{ \pm \frac{1}{2}\right\}} \int_{\mathbb{R}^{6}} \chi_{P}(x, s) \chi_{Q}\left(y, s^{\prime}\right) \frac{1}{|x-y|} \chi_{R}(x, s) \chi_{S}\left(y, s^{\prime}\right) d x d y
$$

defining the antisymmetrized integrals

$$
\langle P Q \| R S\rangle:=\frac{1}{2}(\langle P Q \mid R S\rangle-\langle P Q \mid S R\rangle) .
$$

Next, we rewrite cluster operators $T=\sum_{\mu \in \mathcal{M}} t_{\mu} X_{\mu}$ in terms of creation and annihilation operators: As in Section 2 (iii), we note that for any given $\mu \in \mathcal{M}$ the corresponding determinant $\Psi_{\mu}$ can be obtained from $\Psi_{0}$ by exchanging $r$ indices $I_{1}<\ldots<I_{r} \in$ occ against virtual indices $A_{1}<\ldots<A_{r} \in$ virt, and this defines the operator $X_{\mu}$. It is not hard to see that such $X_{\mu}$ can be expressed in terms of annihilation and creation operators as

$$
X_{\mu}=X_{I_{1}, \ldots, I_{r}}^{A_{1}, \ldots, A_{r}}=a_{A_{1}}^{\dagger} \ldots a_{A_{r}}^{\dagger} a_{I_{1}} \ldots a_{I_{r}} .
$$

Thus, the central part $\mathrm{e}^{-T} H \mathrm{e}^{T}$ of the CC equations can be expressed completely in terms of annihilation and creation operators.

(iii) Termination of the Baker-Campbell-Hausdorff series. The key to prove the missing part of Theorem A.1 is the below Lemma A.3. Before it can be formulated, we need one notation: Let $\mathcal{E}$ be an arbitrary set of annihilation and creation operators, $B=b_{1} \ldots b_{n}$ an operator string, i.e. an operator built of annihilation and creation operators. We denote by

$$
\mathcal{C}_{\mathcal{E}}(B)=\mid\left\{b_{i} \mid 1 \leq i \in \leq N, \quad \exists e \in \mathcal{E} \text { such that }\left[b_{i}, e\right]_{+} \neq 0\right\} \mid
$$

the number of operators in $B$ that do not anticommute with all contained in $\mathcal{E}$. 
Lemma A.3. Let $\mathcal{E}$ be an anticommuting set of creation and annihilation operators, $[e, f]_{+}=0$ for all e, $f \in \mathcal{E}$, and $B, C$ be operator strings for which $B=b_{1} \ldots b_{2 n}$ contains an even number of operators and for which for $C=c_{1} \ldots c_{m}, c_{i} \in \mathcal{E}$ for all $1 \leq i \leq m$. Then, if $\mathcal{C}_{\mathcal{E}}(B)=0$, there holds $[B, C]=0$, and in case $\mathcal{C}_{\mathcal{E}}(B) \geq 1$, we can write $[B, C]=\sum_{i=1}^{n} B_{i}$ with operator strings $B_{i}$ for which $\mathcal{C}_{\mathcal{E}}\left(B_{i}\right) \leq \mathcal{C}_{\mathcal{E}}(B)-1$.

Proof. We proceed by induction over $m$. For $m=1$, there follows by definition of the anticommutator that by swapping $c_{1}$ to the left,

$$
\begin{aligned}
{\left[B, c_{1}\right] } & =b_{1} \ldots b_{2 n} c_{1}-c_{1} b_{1} \ldots b_{2 n} \\
& =(-1)^{2 n} c_{1} b_{1} \ldots b_{2 n}-c_{1} b_{1} \ldots b_{2 n}+\sum_{i=0}^{2 n}(-1)^{i}\left[b_{i}, c_{1}\right]_{+} b_{1} \ldots b_{i-1} b_{i+1} \ldots b_{2 n} .
\end{aligned}
$$

The first two terms cancel. In the sum in the last, we have in each summand either $\left[b_{i}, c_{1}\right]_{+}=0$, or that $\left[b_{i}, c_{1}\right]_{+}=1$ and the operator string $b_{1} \ldots b_{i-1} b_{i+1} \ldots b_{2 n}$ contains one operator less not anticommuting with all operators from $C$. Thus, if $\mathcal{C}_{\mathcal{E}}(B)=0$, we have $\left[b_{i}, c_{1}\right]_{+}=0$ for all $1 \leq i \leq N$, so $\left[B, c_{1}\right]=0$, and if $\mathcal{C}_{\mathcal{E}}(B) \geq 1$, $[B, C]$ is a sum of operator strings $B_{i}$ for which $\mathcal{C}_{\mathcal{E}}\left(B_{i}\right) \leq \mathcal{C}_{\mathcal{E}}(B)-1$. For the induction step, we use the same proceeding for $C=c_{1} \ldots c_{m+1}$ to swap $c_{m+1}$ to the right,

$$
\begin{aligned}
{[B, C] } & =b_{1} \ldots b_{2 n} c_{1} \ldots c_{m+1}-c_{1} \ldots c_{m} c_{m+1} b_{1} \ldots b_{2 n} \\
& =\left[B, C_{m}\right] c_{m+1}+\sum_{i=0}^{2 n}(-1)^{i}\left[c_{m+1}, b_{i}\right]_{+} c_{1} \ldots c_{m} b_{1} \ldots b_{i-1} b_{i+1} \ldots b_{2 n}
\end{aligned}
$$

where we let $C_{m}=c_{1} \ldots c_{m}$. In the case that $\mathcal{C}_{\mathcal{E}}(B)=0$, there follows $\left[B, C_{m}\right] c_{m+1}=0$ by induction hypothesis, and all summands in the second term are also zero because $\left[c_{m+1}, b_{i}\right]_{+}=0$. Thus, $[B, C]=0$. If $\mathcal{C}_{\mathcal{E}}(B) \neq 0$, we observe for the left term that by induction hypothesis, we can write $\left[B, C_{m}\right]$ as a sum of operator strings $B_{i}$ for which $\mathcal{C}_{\mathcal{E}}\left(B_{i}\right) \leq \mathcal{C}_{\mathcal{E}}(B)-1$, so the same holds for $\left[B, C_{m}\right] c_{m+1}$. For the right term, the same argument as in the case $m=1$ gives that each summand can only contain $\mathcal{C}_{\mathcal{E}}(B)-1$ operators that do not commute with all operators $c_{i} \in \mathcal{E}$ (note that the operators from $\mathcal{E}$ anticommute). This completes the proof.

Proof of Theorem A.1. We define

$$
\mathcal{E}:=\left\{a_{I} \mid I \in \text { occ }\right\} \cup\left\{a_{A}^{\dagger} \mid A \in \operatorname{virt}\right\} .
$$

All elements from $\mathcal{E}$ anticommute by (A.3), and all excitation operators $X_{\mu}$ are strings built from elements of $\mathcal{E}$. Using the expression (A.4), we obtain

$$
\begin{aligned}
\mathrm{e}^{-T} H \mathrm{e}^{T}=\sum_{n=0}^{\infty} \frac{1}{n !}[H, T]_{(n)}= & \sum_{n=0}^{\infty} \frac{1}{n !} \sum_{P, Q \in \mathbb{N}} h_{P Q} \quad\left[a_{P}^{\dagger} a_{Q}, \sum_{\mu \in \mathcal{M}} t_{\mu} X_{\mu}\right]_{(n)} \\
& +\sum_{n=0}^{\infty} \frac{1}{n !} \sum_{P, Q, R, S \in \mathbb{N}}\langle P Q \| R S\rangle\left[a_{P}^{\dagger} a_{Q}^{\dagger} a_{R} a_{S}, \sum_{\mu \in \mathcal{M}} t_{\mu} X_{\mu}\right]_{(n)} .
\end{aligned}
$$

For each string $a_{P}^{\dagger} a_{Q}$, there holds $\mathcal{C}_{\mathcal{E}}\left(a_{P}^{\dagger} a_{Q}\right) \leq 2$. We observe that the commutator is linear in its first argument, so iterating Lemma A.3 gives

$$
\mathcal{C}_{\mathcal{E}}\left(\left[\left[a_{P}^{\dagger} a_{Q}, X_{\mu_{1}}\right], X_{\mu_{2}}\right]\right)=0, \quad\left[\left[\left[a_{P}^{\dagger} a_{Q}, X_{\mu_{1}}\right], X_{\mu_{2}}\right], X_{\mu_{3}}\right]=0
$$

for all $\mu_{1}, \mu_{2}, \mu_{3} \in \mathcal{M}$, so

$$
\left[a_{P}^{\dagger} a_{Q}, \sum_{\mu \in \mathcal{M}} t_{\mu} X_{\mu}\right]_{(n)}=0
$$

follows for $n \geq 3$. To the iterated commutators $\left[a_{P}^{\dagger} a_{Q}^{\dagger} a_{R} a_{S}, \sum_{\mu \in \mathcal{M}} t_{\mu} X_{\mu}\right]_{(n)}$, an analogous argument applies to show that those of order $n \geq 5$ must give zero contributions, so the proof is finished. 
Remark A.4. We remark that the representation (A.2) coincides with the Taylor expansion of the $C^{\infty}$-function $g(T)=\mathrm{e}^{-T} A \mathrm{e}^{T}$ at $T=0$ : more generally, it is given for arbitrary $S \in B\left(\mathbb{H}^{1}\right)$ by $g(S+T)=\mathrm{e}^{-T-S} A \mathrm{e}^{T+S}=$ $\sum_{i=0}^{4} 1 / n !\left[\mathrm{e}^{-S} A \mathrm{e}^{S}, T\right]_{(n)}$. In particular, this implies $f^{(5)} \equiv 0$ for the CC function.

(iv) Evaluation of the iterated commutators. To apply solvers like, e.g., the below simple inexact Newton's method to solve the root problem (2.28), the Coupled Cluster function and thus, by Theorem A.1, the expression

$$
f(t)=\sum_{n=0}^{4} \frac{1}{n !}\left\langle\Psi_{\mu},[H, T]_{(n)} \Psi_{0}\right\rangle=\sum_{n=0}^{4} \frac{1}{n !}\left\langle\Psi_{0}, X_{\mu}^{\dagger}[H, T]_{(n)} \Psi_{0}\right\rangle
$$

has to be evaluated for all $\mu \in \mathcal{M}$. This is a nontrivial task, and for sake of brevity, we only sketch the canonical proceeding here and refer the reader to the comprehensive introduction [28] and the references given therein for deeper insight. To evaluate (A.10), Wick's theorem, proven, e.g., in [60] and based on the anticommutator relations (A.3), is the fundamental tool used to successively reorder the operator strings contained in $X_{\mu}^{\dagger}[H, T]_{(n)}$ to sums of normal-ordered strings (i.e. strings where for $\mathcal{E}$ defined by (A.9), all operators $b \in \mathcal{E}$ are to the left of all operators $b \notin \mathcal{E}$ ). Normal-ordered strings give a zero contribution to (A.10), and the remainder term is a sum of so called "fully contracted terms", containing no annihilation/creation operators anymore as a result of various anticommutators arising in the ordering process. The task is now to determine all of those admissible fully contracted terms that yield a nonzero distribution to (A.10), and this boils down to choosing the right indices of matrix elements of $H$ and of up to four different cluster amplitudes $t_{\nu}$ that contribute to each $\mu$-th component of $f(t)$ according to certain rules. This combinatorial task is nontrivial, and especially tedious to implement because the fully contracted terms feature various combinations of signs arising from the anticommutation laws. Therefore, an approach popularized in [49] is normally used to rewrite the equations as diagrams (see also [28]); then, computable expressions are derived from these diagrams by a fixed set of rules, either by hand or automatedly as, e.g., in $[5,45]$. To give the reader an impression of the resulting equations, we depicted the update equations for the energy and the $T_{1}$ - $/ T_{2}$-amplitudes for the frequently used CCSD procedure in Figure 5. In this, the coefficients $f_{P Q}$ are those determined by the according one-particle operator $F$. Evaluation for the "doubles" amplitudes $f(t)_{I J}^{A B}$ is an $N^{6}$ step if one supposes that the number of virtual orbitals in the chosen discretisation is proportional to $N$. Note the most expensive summation step (but not the only $N^{6}$ step) is given by the term $\frac{1}{2} \sum_{C D}\langle A B \| C D\rangle t_{I J}^{C D}$ contributing to $f(t)_{I J}^{A B}$ (see the second line). Denoting the number of virtual orbitals by $V$, this step consists of a summation over $V^{2}$ terms for each of the $N^{2} V^{2}$ amplitudes, so that the evaluation of this contribution is a step of complexity $N^{2} V^{4}$, which usually consumes (due to the constants entering by $V=C \cdot N$ ) about $70-90 \%$ of the overall computation time. Efficient factorization of the CC equations is another topic of its own, cf., e.g., the references in [28].

(iv) Newton's method and fixed point iteration for the $\mathrm{CC}$ function. To solve the CC root equation (2.28), it is common practice to use an inexact Newton's method or a related fixed point scheme with the (lifted, shifted) Fock matrix as approximate Jacobian. We obtain some results concerning these methods, concluding this present section.

Corollary A.5 (Convergence of a quasi-Newton method).

Let $P: \mathbb{V} \rightarrow \mathbb{V}^{\prime}$ be any linear bounded coercive linear mapping. If $E^{*}$ is a simple eigenvalue and $\Psi_{0}$ is close enough to $\underline{\Psi}$, there is an $\alpha>0$ such that the damped inexact Newton's method

$$
t_{n+1}=t_{n}-\alpha P^{-1} f\left(t_{n}\right)
$$

with starting value $\Psi_{0}$ converges to $\underline{\Psi}$. If $\left\|D f\left(t^{*}\right)-P\right\|$ is small enough, $\alpha=1$ is a possible choice.

The proof is identical with that for the finite dimensional case, which can be derived, e.g., from Theorem 8.2.2 in [30], so it is omitted. We only note that also in the convergence estimates for Newton's method, the decisive constants are the local Lipschitz constant $L$ and the monotonicity constant $\gamma$ of the CC function. 


$$
\begin{aligned}
& E(t)=\left\langle\Psi_{0}, H \Psi_{0}\right\rangle+\sum_{I A} f_{I A} t_{I}^{A}+\frac{1}{4} \sum_{I J A B}\langle I J \| A B\rangle t_{I J}^{A B}+\frac{1}{2} \sum_{I J A B}\langle I J \| A B\rangle t_{I}^{A} t_{J}^{B}, \\
& f(t)_{I}^{A}=f_{I A}+\sum_{C} f_{A C} t_{I}^{C}-\sum_{K} f_{K I} t_{K}^{A}+\sum_{K C}\langle K A \| C I\rangle t_{C}^{K}+\sum_{K C} f_{K C} t_{I K}^{A C} \\
& +\frac{1}{2} \sum_{K C D}\langle K A \| C D\rangle t_{K I}^{C D}-\frac{1}{2} \sum_{K L C}\langle K L \| C I\rangle t_{K L}^{C A}-\sum_{K C} f_{K C} t_{I}^{C} t_{K}^{A}-\sum_{K L C}\langle K L \| C I\rangle t_{K}^{C} t_{L}^{A} \\
& +\sum_{K C D}\langle K A \| C D\rangle t_{K}^{C} t_{I}^{D}-\sum_{K L C D}\langle K L \| C D\rangle t_{K}^{C} t_{I}^{D} t_{L}^{A}+\sum_{K L C D}\langle K L \| C D\rangle t_{C}^{K} t_{L I}^{D A} \\
& -\frac{1}{2} \sum_{K L C D}\langle K L \| C D\rangle t_{K I}^{C D} t_{L}^{A}-\frac{1}{2} \sum_{K L C D}\langle K L \| C D\rangle t_{K L}^{C A} t_{I}^{D} \\
& f(t) \underset{I J}{A B}=\langle I J \| A B\rangle+\sum_{C}\left(f_{B C} t_{I J}^{A C}-f_{A C} t_{I J}^{B C}\right)-\sum_{K}\left(f_{K} t_{I K}^{A B}-f_{K I} t_{J K}^{A B}\right) \\
& +\frac{1}{2} \sum_{K L}\langle K L \| I J\rangle t_{K L}^{A B}+\frac{1}{2} \sum_{C D}\langle A B \| C D\rangle t_{I J}^{C D}+P(I J) P(A B) \sum_{K C}\langle K B \| C J\rangle t_{I K}^{A C} \\
& +P(I J) \sum_{C}\langle A B \| C J\rangle t_{I}^{C}-P(A B) \sum_{K}\langle K B \| I J\rangle t_{A}^{K} \\
& +\frac{1}{2} P(I J) P(A B) \sum_{K L C D}\langle K L \| C D\rangle t_{I K}^{A C} t_{L J}^{D B}+\frac{1}{4} \sum_{K L C D}\langle K L \| C D\rangle t_{I J}^{C D} t_{K L}^{A B} \\
& +\frac{1}{2} P(A B) \sum_{K L C D}\langle K L \| C D\rangle t_{I J}^{A C} t_{K L}^{B D}-\frac{1}{2} P(I J) \sum_{K L C D}\langle K L \| C D\rangle t_{I K}^{A B} t_{J L}^{C D} \\
& +\frac{1}{2} P(A B) \sum_{K L}\langle K L \| I J\rangle t_{K}^{A} t_{L}^{B}+\frac{1}{2} P(I J) \sum_{C D}\langle A B \| C D\rangle t_{I}^{C} t_{J}^{D} \\
& \text { - } P(I J) P(A B) \sum_{K C}\langle K B \| I C\rangle t_{K}^{A} t_{J}^{C}+P(A B) \sum_{K C} f_{K C} t_{K}^{A} t_{I J}^{B C} \\
& +P(I J) \sum_{K C} f_{K C} t_{I}^{C} t_{J K}^{A B}-P(I J) \sum_{K L C}\langle K L \| C I\rangle t_{K}^{C} t_{L J}^{A B} \\
& +P(A B) \sum_{K C D}\langle K A \| C D\rangle t_{K}^{C} t_{I J}^{D B}+P(I J) P(A B) \sum_{K C D}\langle A K \| D C\rangle t_{I}^{D} t_{J K}^{B C} \\
& +P(I J) P(A B) \sum_{K L C}\langle K L \| I C\rangle t_{L}^{A} t_{J K}^{B C}+\frac{1}{2} P(I J) \sum_{K L C}\langle K L \| C J\rangle t_{I}^{C} t_{K L}^{A B} \\
& -\frac{1}{2} P(A B) \sum_{K C D}\langle K B \| C D\rangle t_{K}^{A} t_{I J}^{C D}+\frac{1}{2} P(I J) P(A B) \sum_{K L C}\langle K B \| C D\rangle t_{I}^{C} t_{K}^{A} t_{J}^{D} \\
& +\frac{1}{2} P(I J) P(A B) \sum_{K L C}\langle K L \| C J\rangle t_{I}^{C} t_{K}^{A} t_{L}^{B}-P(I J) \sum_{K L C D}\langle K L \| C D\rangle t_{K}^{C} t_{I}^{D} t_{L J}^{A B} \\
& \text { - P(AB) } \sum_{K L C D}\langle K L \| C D\rangle t_{K}^{C} t_{L}^{A} t_{I J}^{D B}-\frac{1}{4} P(I J) \sum_{K L C D}\langle K L \| C D\rangle t_{I}^{C} t_{J}^{D} t_{K L}^{A B} \\
& +\frac{1}{4} P(A B) \sum_{K L C D}\langle K L \| C D\rangle t_{K}^{A} t_{L}^{B} t_{I J}^{C D}+P(I J) P(A B) \sum_{K L C D}\langle K L \| C D\rangle t_{I}^{C} t_{L}^{B} t_{K J}^{A D} \\
& +\frac{1}{4} P(I J) P(A B) \sum_{K L C D}\langle K L \| C D\rangle t_{I}^{C} t_{K}^{A} t_{J}^{D} t_{L}^{B}
\end{aligned}
$$

Figure 1. The CCSD equations for the CC energy $E(t)$ and for the $T_{1}, T_{2}$ amplitudes $f(t)_{I}^{A}, f(t)_{I, J}^{A, B},(I, J \in$ occ, $A, B \in$ virt $)$. In this, $P(I J) f(I, J):=f(I, J)-f(J, I)$.

For computational purposes, let us finally consider the discrete setting. In this case it is common practice to choose the Hartree-Fock determinant $\Psi_{H F}$ as reference $\Psi_{0}$ and the eigenfunctions $\varphi_{i}$ of the discrete Fock operator as a basis set (molecular orbitals or canonical orbitals). We may decompose the Full CI Hamiltonian $\mathbf{H}$ into the (lifted, shifted) Fock operator $\mathbf{F}$ from the preliminary self-consistent Hartree-Fock computation - a diagonal matrix if canonical orbitals are chosen - and the remainder $\mathbf{U}$, a two-particle operator called fluctation potential, viz., $\mathbf{H}=\mathbf{F}+\mathbf{U}$. It can be shown [82] that in this case, for any discrete coefficient vector $\mathbf{t}=\left(t_{\mu}\right)_{\mu \in \mathcal{M}_{d}}$,

$$
\hat{\mathbf{P}}(\mathbf{t}):=\mathrm{e}^{-T(\mathbf{t})} \mathbf{F} \mathrm{e}^{T(\mathbf{t})}=\left(\mathbf{F}-\Lambda_{0}\right) T(\mathbf{t})=\left(\operatorname{diag}\left(\lambda_{\mu}\right)\right) T(\mathbf{t}),
$$

where, denoting the one-particle Hartree Fock energies by $\epsilon_{P}$, i.e., $\mathbf{F} \chi_{P}=\epsilon_{P} \chi_{P}$, we used the notation $\Lambda_{0}=$ $\sum_{I=1}^{N} \epsilon_{I}$ for the sum of the eigenvalues of the occupied orbitals, and $\lambda_{\mu}>0$ for all $\mu \in \mathcal{M}_{d}$ is calculated from differences of the eigenvalues. Note that $\hat{\mathbf{P}}$ does not depend on $\mathbf{t}$ in this setting. does not depend on $\mathbf{t}$ anymore 
in this setting. Then the root of the Coupled Cluster function $\mathbf{f}$ corresponds to a fixed point of the function $\mathrm{g}: \mathbb{V}_{d} \rightarrow \mathbb{V}_{d}$

$$
\mathbf{t} \mapsto \mathbf{g}(\mathbf{t}):=\hat{\mathbf{P}}^{-1}\left(\left\langle\Psi_{\mu}, \mathrm{e}^{-T(\mathbf{t})} \mathbf{U e}^{T(\mathbf{t})} \Psi_{0}\right\rangle\right)_{\mu \in \mathcal{M}_{d}}
$$

Let us finally note that a fixed point iteration for the function $\mathbf{g}$ is a common iteration scheme in practice. It converges if $\mathbf{g}$ is (locally) a contraction, which is the case if and only of the conditions of Theorem 3.7 (applied to the discrete version of $f$ ) are fulfilled. This again emphasizes the perturbational nature of the practical Coupled Cluster approach.

\section{REFERENCES}

[1] A. Anantharaman and E. Cancès, Existence of minimizers for Kohn-Sham models in quantum chemistry. Ann. Institut Henri Poincaré, Non Linear Anal. 26 (2009) 2425.

[2] S. Agmon, Lectures on exponential decay of solutions of second-order elliptic equations. Princeton University press, Princeton (1982).

[3] H.W. Alt, Lineare Funktionalanalysis, Auflage. Springer, Berlin 5 (2006).

[4] J. Arponen, Variational principles and linked-cluster exp S expansions for static and dynamic many-body problems. Ann. Phys. 151 (1983) 311.

[5] A.A. Auer and G. Baumgärtner, Automatic Code Generation for Many-Body Electronic Structure Methods: The tensor contraction engine. Molecul. Phys. 104 (2006) 211.

[6] I. Babuska and J.E. Osborn, Finite Element-Galerkin Approximation of the Eigenvalues and Eigenvectors of Selfadjoint Problems. Math. Comput. 52 (1989) 275-297.

[7] V. Bach, E.H. Lieb, M. Loss and J.P. Solovej, There are no unfilled shells in unrestricted Hartree-Fock theory. Phys. Rev. Lett. 72 (1994) 2981.

[8] N.B. Balabanova and K.A. Peterson, Basis set limit electronic excitation energies, ionization potentials, and electron affinities for the 3d transition metal atoms: Coupled cluster and multireference methods. J. Chem. Phys. 125 (2006) 074110.

[9] W. Bangerth and R. Rannacher, Adaptive finite element methods for differential equations. Birkhäuser (2003).

[10] R.J. Bartlett, Many-body perturbation theory and coupled cluster theory for electronic correlation in molecules. Ann. Rev. Phys. Chem. 32 (1981) 359.

[11] R.J. Bartlett and M. Musial, Coupled-cluster theory in quantum chemistry. Rev. Mod. Phys. 79 (2007) 291.

[12] R.J. Bartlett and G.D. Purvis, Many-body perturbation theory, coupled-pair many-electron theory, and the importance of quadruple excitations for the correlation problem. Int. J. Quantum Chem. 14 (1978) 561.

[13] R. Becker and R. Rannacher, An optimal control approach to error estimation and mesh adaptation in finite element methods. Acta Numerica 2000. Edited by A. Iserles. Cambridge University Press (2001) 1.

[14] U. Benedikt, M. Espig, W. Hackbusch and A.A. Auer, A new Approach for Tensor Decomposition in Electronic Structure Theory (submitted).

[15] D.E. Bernholdt and R.J. Bartlett, A Critical Assessment of Multireference-Fock Space CCSD and Perturbative Third-Order Triples Approximations for Photoelectron Spectra and Quasidegenerate Potential Energy Surfaces. Adv. Quantum Chemist. 34 (1999) 261.

[16] R.F. Bishop, An overview of coupled cluster theory and its applications in physics. Theor. Chim. Acta 80 (1991) 95.

[17] M. Born and R. Oppenheimer, Zur Quantentheorie der Molekeln. Ann. Phys. 389 (1927) 457.

[18] E. Cancès, R. Chakir and Y. Maday, Numerical Analysis of Nonlinear Eigenvalue Problems J. Scientific Comput. 45 (2010) 90. DOI : $10.1007 / \mathrm{s} 10915-010-9358-1$.

[19] P. Cársky, J. Paldus and J. Pittner, Recent Progress in Coupled Cluster Methods, Theory and Applications. In vol. 44 of series: Challenges Adv. Comput. Chem. Phys. Springer (2010).

[20] T. Chan, W.J. Cook, E. Hairer, J. Hastad, A. Iserles, H.P. Langtangen, C. Le Bris, P.L. Lions, C. Lubich, A.J. Majda, J. McLaughlin, R.M. Nieminen, J.T. Oden, P. Souganidis and A. Tveito, Encyclopedia Appl. Comput. Math. Springer. To appear (2013).

[21] O. Christiansen, Coupled cluster theory with emphasis on selected new developments. Theor. Chem. Acc. 116 (2006) 106.

[22] P.G. Ciarlet and J.L. Lions, Handbook of Numerical Analysis, Volume II: Finite Element Methods (Part I). Elsevier (1991).

[23] P.G. Ciarlet and C. Lebris, Handbook of Numerical Analysis, Volume X: Special Volume. Computational Chemistry. Elsevier (2003).

[24] J. Čížek, Origins of coupled cluster technique for atoms and molecules. Theor. Chim. Acta 80 (1991) 91.

[25] F. Coerster, Bound states of a many-particle system. Nucl. Phys. 7 (1958) 421.

[26] F. Coerster and H. Kümmel, Short range correlations in nuclear wave functions. Nucl. Phys. 17 (1960) 477.

[27] Computational Chemistry Comparison and Benchmark Data Base, National Institute of Standards and Technology. Available on www.cccbdb.nist.gov.

[28] T.D. Crawford and H.F. Schaeffer III, An introduction to coupled cluster theory for computational chemists. Rev. Comput. Chem. 14 (2000) 33. 
[29] Dalgaard and H.J. Monkhorst, Some aspects of the time-dependent coupled-cluster approach to dynamic response functions. Phys. Rev. A 28 (1983) 1217.

[30] J.E. Dennis Jr. and R.B. Schnabel, Numerical Methods for Unconstrained Optimization and Nonlinear Equations. SIAM, Philadelphia (1996).

[31] P.A.M. Dirac, Quantum Mechanics of Many-Electron Systems. Proc. of Royal Soc. London, Series A CXXIII (1929) 714.

[32] R.M. Dreizler and E.K.U. Gross, Density functional theory. Springer (1990).

[33] E. Emmrich, Gewöhnliche und Operator-Differentialgleichungen, Vieweg (2004).

[34] H.J. Flad, R. Schneider and T. Rohwedder, Adaptive methods in Quantum Chemistry. Zeitsch. f. Phys. Chem. 224 (2010) $651-670$

[35] V. Fock, Konfigurationsraum und zweite Quantelung. Z. Phys. 75 (1932) 622.

[36] G. Friesecke and B.D. Goddard, Explicit large nuclear charge limit of electronic ground states for Li, Be, B, C, N, O, F, Ne and basic aspects of the periodic table. SIAM J. Math. Anal. 41 (2009) 631-664.

[37] H. Gajewski, K. Gröger and K. Zacharias, Nichtlineare Operatorgleichungen und Operatordifferentialgleichungen, Akademie Verlag (1974).

[38] S.R. Gwaltney and M. Head-Gordon, A second-order correction to singles and doubles coupled-cluster methods based on a perturbative expansion of a similarity-transformed Hamiltonian 323 (2000) 2128.

[39] S.R. Gwaltney, C.D. Sherrill, M. Head-Gordon and A.I. Krylov, Second-order perturbation corrections to singles and doubles coupled-cluster methods: General theory and application to the valence optimized doubles model. J. Chem. Phys. 113 (2000) $3548-3560$.

[40] W. Hackbusch, Elliptic Differential Equations, vol. 18. of SSCM. Springer (1992),

[41] C. Hampel and H.-J. Werner, Local treatment of electron correlation in coupled cluster theory. J. Chem. Phys. 104 (1996) 6286 .

[42] T. Helgaker and P. Jørgensen, Configuration-interaction energy derivatives in a fully variational formulation. Theor. Chim. Acta $\mathbf{7 5}$ (1989) 111127

[43] T. Helgaker, P. Jørgensen and J. Olsen, Molecular Electronic-Structure Theory. John Wiley \& Sons (2000).

[44] T. Helgaker, W. Klopper and D.P. Tew, Quantitative quantum chemistry. Mol. Phys. 106 (2008) 2107.

[45] S. Hirata, Tensor contraction engine: Abstraction and automated parallel implementation of Configuration-Interaction, Coupled-Cluster, and Many-Body perturbation theories. J. Phys. Chem. A 46 (2003) 9887.

[46] W. Hunziker and I.M. Sigal, The quantum N-body problem. J. Math. Phys. 41 (2000) 6.

[47] W. Klopper, F.R. Manby, S. Ten-no and E.F. Vallev, R12 methods in explicitly correlated molecular structure theory. Int. Rev. Phys. Chem. 25 (2006) 427.

[48] P. Knowles, M. Schütz and H.-J. Werner, Ab Initio Methods for Electron Correlation in Molecules, Modern Methods and Algorithms of Quantum Chemistry, vol. 3 of Proceedings, Second Edition, edited by J. Grotendorst. John von Neumann Institute for Computing, Jülich, NIC Series, ISBN 3-00-005834-6 (2000) 97-179.

[49] S.A. Kucharsky and R.J. Bartlett, Fifth-order many-body perturbation theory and its relationship to various coupled-cluster approaches. Adv. Quantum Chem. 18 (1986) 281.

[50] W. Kutzelnigg, Error analysis and improvement of coupled cluster theory, Theoretica Chimica Acta 80 (1991) 349.

[51] H. Kümmel, Compound pair states in imperfect Fermi gases. Nucl. Phys. 22 (1961) 177.

[52] H. Kümmel, K.H. Lührmann and J.G. Zabolitzky, Many-fermion theory in expS- (or coupled cluster) form. Phys. Reports 36 (1978) 1.

[53] S. Kvaal, Ab initio quantum dynamics using coupled-cluster, to appear in J. Chem. Phys. (2012).

[54] T.J. Lee, Comparison of the T1 and D1 diagnostics for electronic structure theory: a new definition for the open-shell D1 diagnostic. Chem. Phys. Lett. 372 (2003) 362-367.

[55] T.J. Lee and G.E. Scuseria, Achieving chemical accuracy with Coupled Cluster methods, in Quantum Mechanical Electronic Structure Calculations with Chemical Accuracy, edited by S.R. Langhof. Kluwer Academic Publishers, Dordrecht (1995) 47.

[56] T.J. Lee and P.R. Taylor, A diagnostic for determining the quality of single-reference electron correlation methods. Int. J. Quantum Chem. Symp. 23 (1989) 199-207.

[57] X. Li and J. Paldus, Dissociation of N2 triple bond: a reduced multireference CCSD study. Chem. Phys. Lett. 286 12 (1998) $145-154$.

[58] E.H. Lieb and B. Simon, The Hartree-Fock Theory for Coulomb Systems. Commun. Math. Phys. 53 (1977) 185.

[59] E.H. Lieb, Bound on the maximum negative ionization of atoms and molecules. Phys. Rev. A 29 (1984) 3018.

[60] I. Lindgren and J. Morrison, Atomic Many-body Theory. Springer (1986).

[61] P.L. Lions, Solution of the Hartree Fock equation for Coulomb Systems. Commun. Math. Phys. 109 (1987) 33.

[62] C. Lubich, From Quantum to Classical Molecular Dynamics: Reduced methods and Numerical Analysis. Zürich Lect. Adv. Math. EMS (2008).

[63] D.I. Lyakh, V.V. Ivanov and L. Adamowicz, State-specific multireference complete-active-space coupled-cluster approach versus other quantum chemical methods: dissociation of the N2 molecule. Mol. Phys. 105 (2007) 1335-1357.

[64] D.I. Lyakh and R.J. Bartlett, An adaptive coupled-cluster theory: @CC approach. J. Chem. Phys. 133 (2010) 244112.

[65] F. Neese, A. Hansen and D.G. Liakos, Efficient and accurate approximations to the local coupled cluster singles doubles method using a truncated pair natural orbital basis. J. Chem. Phys. 131 (2009) 064103. 
[66] U.S. Mahapatra, B. Datta and D. Mukherjee, A size-consistent state-specific multireference coupled cluster theory: Formal developments and molecular applications. J. Chem. Phys. 110 (1999) 6171-6188.

[67] M. Nooijen, K.R. Shamasundar and D. Mukherjee, Reflections on size-extensivity, size-consistency and generalized extensivity in many-body theory. Molecular Phys. 103 (2005) 2277.

[68] J. Paldus, Coupled Cluster Theory, in Methods Comput. Molec. Phys., edited by S. Wilson and G.F.H. Diercksen. Plenum. New York (1992) 99.

[69] J. Paldus, M. Takahashi and B.W.H. Cho, Degeneracy and coupled-cluster Approaches 26 (1984) $237-244$.

[70] R.G. Parr and W. Yang, Density-Functional Theory of Atoms and Molecules. Oxford University Press (1994).

[71] A. Persson, Bounds for the discrete part of the spectrum of a semibounded Schrödinger operator. Math. Scand. 8 (1960) 143.

[72] P. Piecuch, N. Oliphant and L. Adamowicz, A state-selective multireference coupled-cluster theory employing the singlereference formalism. J. Chem. Phys. 99 (1993) 1875.

[73] P. Piecuch, K. Kowalski, P.-D. Fan and I.S.O. Pimienta, New alternatives for electronic structure calculations: Renormalized, extended, and generalized coupled-cluster theories, in vol. 12 of Progr. Theoret. Chemist. Phys., edited by J. Maruani, R. Lefebvre, E. Brändas. Kluwer, Dordrecht (2003) 119-206.

[74] J. Pousin and J. Rapaz, Consistenct, stability, a priori and a posteriori estimates for Petrov-Galerkin methods applied to nonlinear problems. Num. Math. 69 (1994) 213-231.

[75] K. Raghavachari, G.W. Trucks, J.A. Pople and M. Head-Gordon, A fifth-order perturbation comparison of electronic correlation theories. Chem. Phys. Lett. 157 (1989) 479.

[76] M. Reiher, A Theoretical Challenge: Transition-Metal Compounds, Chimia 63 (2009) 140-145.

[77] M. Reed and B. Simon, Methods of Modern Mathematical Physics IV - Analysis of operators. Academic Press (1978).

[78] T. Rohwedder, An analysis for some methods and algorithms of Quantum Chemistry, Ph.D. thesis, TU Berlin, available at http://opus.kobv.de/tuberlin/volltexte/2010/2852/ (2010).

[79] T. Rohwedder, The continuous Coupled Cluster formulation for the electronic Schrödinger equation, submitted to M2AN.

[80] W. Rudin, Functional Analysis. Tat McGraw \& Hill Publishing Company, New Delhi (1979).

[81] Y. Saad, J.R. Chelikowsky and S.M. Shontz, Numerical Methods for Electronic Structure Calculations of Materials. SIAM Rev. 52 (2010) 1.

[82] R. Schneider, Analysis of the projected Coupled Cluster method in electronic structure calculation. Num. Math. 113 (2009) 433.

[83] M. Schütz and H.-J. Werner, Low-order scaling local correlation methods. IV. Linear scaling coupled cluster (LCCSD). J. Chem. Phys. 114 (2000) 661.

[84] B. Simon, Schrödinger operators in the 20th century. J. Math. Phys. 41 (2000) 3523.

[85] A. Szabo and N.S. Ostlund, Modern Quantum Chemistry. Dover Publications Inc. (1992).

[86] D.J. Thouless, Stability conditions and nuclear rotations in the Hartree-Fock theory. Nuclear Phys. 21 (1960) 225.

[87] J. Wloka, Partial differential equations. Cambridge University Press, reprint (1992).

[88] H. Yserentant, Regularity and Approximability of Electronic Wave Functions, in vol. 2000 of Lect. Notes Math. Ser. SpringerVerlag (2010).

[89] E. Zeidler, Nonlinear Functional Analysis and Its Applications, Part II B: Nonlinear Monotone Operators. Springer (1990).

[90] G.M. Zhislin, Discussion of the spectrum of Schrödinger operator for systems of many particles. Trudy Mosov. Mat. Obshch. 9 (1960) 81-128. 